The abstracts are only available online, free of charge, under www.karger.com/journals/bpu/bpu_bk.htm

\title{
4th Basic Multidisciplinary Hemodialysis Access Course
}

November 8-9, 2001, Rome, Italy

\section{Abstracts}

Guest Editor

Salvatore Di Giulio, Rome, Italy

\section{Contents}

Preoperative Vascular Assessment

Abstracts 1-4

Creation of Vascular Access

Abstracts 5-9

Vascular Access Complications

Abstracts 10-12, 21-31

Treatment of Stenosis and Thrombosis

Abstracts 13-20 


\section{Preoperative Vascular Assessment}

Chairmen: P. Bourquelot, M. Malovrh

\section{The Clinical Examination before Creation of Vascular Access}

Thierry Pourchez

Clinique Ambroise Paré, Béthune, France

The clinical examination is always the first necessary step to draw an operating indication.

\section{Conditions of the Examination}

This must be carried out in good conditions, on a patient feeling safe and in a warm room. Fear and cold have vasoconstrictor effects. One should not hesitate to carry this examination out again before the operation, when the patient is in a warm bed, or a bit later, when a brachial plexus block has induced a significant vasodilatation. The upper limbs are undressed to the shoulders. If the patient is cold, the hand and forearm can be plunged into a washbasin of warm water, which will induce a quick vasodilatation. The presence of subcutaneous oedema or haematoma requires another later examination in better conditions.

\section{Questioning and Case History}

The questioning and different files enable one to find the patient's main medical, surgical and vascular access history. It is necessary to know where venipunture and cannulation have been made in the past, and if central venous catheters or drums had been placed. Normally the bilateral venous tree should already have been protected. It is also necessary to know the level of renal insufficiency, and of any possible allergies, especially to iodine.

Examination without Tourniquet: Inspection: This allows one to see scars related to former vascular access creation, central venous access, various wounds or operations, etc. Phlebitis, or catheters in major veins of the upper limb can bee observed, even in a patient sent by nephrologists! In certain patients, the veins stand out without a tourniquet. On the other hand, obesity impairs the quality of the examination. A collateral circulation of the shoulder must be searched. Palpation and Auscultation: The character of the brachial, radial and ulnar pulses are determined. A hard and non pulsatile artery is usually the consequence of calcifications, especially in diabetic patients. The ulnar pulse is more difficult to find, because the artery is smaller and deeper than the radial artery. A weak or absent humeral pulse is usually due to a proximal arterial obstruction with a difference of pressure between both upper extremities, precluding vascular access creation. But it can be only the result of major calcifications. The high division of the humeral artery can give two different pulses at the elbow, one of which is more superficial. The specific compresion of one of them, if possible, may stop one pulse at the wrist. The Allen test is of no interest, because a single artery at the wrist can feed the hand and a fistula, if its quality is good enough. It is not at all predicting post-operative ischemia. Veins with recent thrombosis are hard and roll under the finger. They may be painful. The sharp auscultation can reveal an old fistula, but especially a bruit on the subclavian or the axillary artery, relating to an arterial stenosis.

Examination with a Tourniquet: The tourniquet is applied high up the upper limb. Movements of flexing and extension of the fingers allows the veins to engorge. They are soft under the fingers, and can be flattened easily. The most important are the cephalic and the basilic veins. Raising of the upper limb before the tourniquet retrieval allows observation of the quality of the venous outflow. Normally the veins empty very quickly. If there is a short obstruction in the venous drainage the vein empties in a few seconds or more.

Radiography of the Soft Tissues: It reveals easily calcifications of the arterial tree.

\section{Conclusion}

The physical examination is always the first step before creating a vascular access. Other methods are useful in selected cases, when this examination is inadequate.

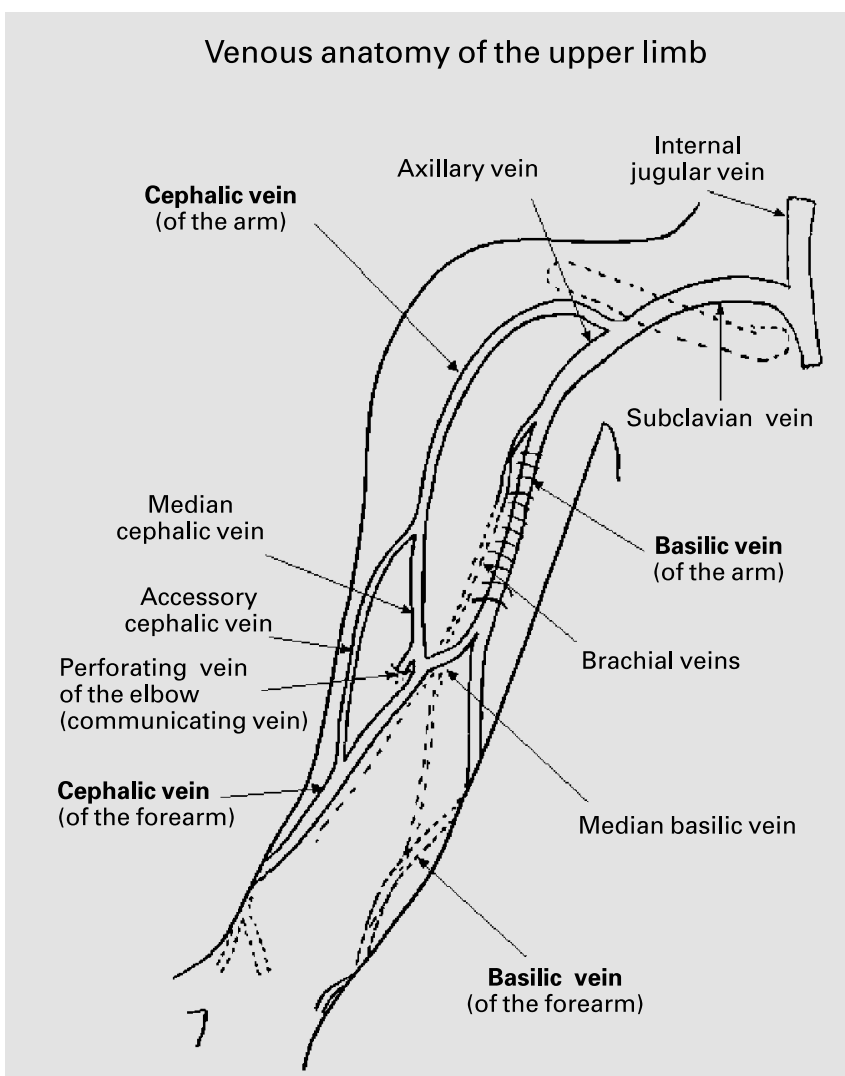

The two major superficial veins are the cephalic and the basilic

\section{KARGER}

Fax + 41613061234

E-Mail karger@karger.ch

www. karger.com
(C) 2002 S. Karger AG, Basel

0253-5068/02/0020-0000\$18.50/0

Accessible online at:

www.karger.com/journals/bpu 
2

\section{Venous Preservation in Predialysis and Early Referral}

\author{
J . Pengloan \\ Unité d'Hémodialyse Chronique, CHU Bretonneau, Tours, \\ France
}

Native arteriovenous (AV) distally created is the gold standard for vascular access (VA) for haemodialysis. Complications of central venous catheters (CVC) are well-known: high rate of infections, stenosis or thrombosis of the host-vein (up to $50 \%$ of stenosis have been reported after subclavian catheterisms) which compromise the creation of peripheral VA on the homolateral limb. Nevertheless a high proportion of patients initiates hemodialysis treatment by a CVC because $\mathrm{AV}$ had not been previously created or was not mature; this situation was observed in $68 \%$ of incident patients in the CHOICE Study in USA and $48 \%$ of incident patients in Catalonia. Two mains reasons explain this situation: late referral of patients to nephrologists and lack of venous preservation. A close relation is observed between referral time to the nephrologist and proportion of patients using a VA at the initiation of haemodialysis therapy: 10\% for those referred less than 1 month to $32 \%$ for those referred 1 to 4 months and $46 \%$ for those referred greater than 12 months before the initiation of hemodialysis therapy (CHOICE Study). An aggressive policy of venous preservation has to be undertaken early before beginning renal replacement therapy (including peritoneal dialysis and renal transplantation). The most important points are: venipunctures made on the dorsum of hands or through the fistula in transplanted patients, radiological interventional procedures instead of surgery for maintaining patency of created VA, ultrasound-guided and not external landmark-guided technique cannulation of central veins, avoid to have a stent obstructive for an important vein such as internal jugular vein. Subclavian route has to be prohibited even for pace-makers or implantable devices. In emergency the cannulation of the femoral vein with a soft silicone catheter has to be preferred to the internal jugular route; this catheter can be left in place during several days or weeks while waiting for a peripheral VA to mature. The preservation of the venous system is a challenge which involves patients, nurses, nephrologists, surgeons and radiologists.

\section{3}

\section{Ultrasound in Vascular Access for Hemodialysis}

Marko Malovrh

Department of Nephrology, University Medical Center

Ljubljana, Slovenia

Background: Adequate blood flow through an arteriovenous fistula (AVF) is essential for adequate hemodialysis in the patients with end stage renal disease (ESRD). The most frequent problem with AVF are primary failure and unsuccessful maturation with inadequate blood flow during hemodialysis treatment. The risk factor for primary failure is not well established, although the quality of vessels is thought to play an important role. One of the most important predictors of successful AVF development is the ability of the venous and arterial vessels to dilate under the influence of the increased shear rates - vessel remodeling. The pre-operative physical examina- tion of the patients' forearm venous and arterial vessels include the quality of the arterial pulse and inspection of the vein with a tourniquet to induce venous congestion. Patients with advanced atherosclerosis and peripheral vascular disease such as diabetics, elderly and smokers are commonly found to have inadequate arterial pulse for AVF surgery. Duplex scanning is a promising method to establish certain morphological and functional parameters of peripheral blood vessels that is non-invasive and safe. This method has been recently used to visualize and measure arterial and venous vessel diameters, and has shown a good correlation between pre-operative determination and peri-operative findings. The ability of the vein to dilate after AVF construction is also important for AVF patency after surgery and for adequate AVF maturation. To determine the ability of the vein to dilate properly increase of the internal vein's diameter after proximal vein compression and to show the continuity of the shape of Doppler vein signal (DVS) could be used. Outflow stenosis could be detected by pre-operative duplex sonography. To determine if there is a disturbance of venous outflow at increased venous return with deep breath (respiratory filling) could be used. In addition, Doppler waveform at the reactive hyperemia, induced by opening of the clenched fist for two minutes changes from threephasic highresistance flow (peripheral resistance for peripheral arteries is normal high) to biphasic low-resistance flow (peripheral resistance become low for short time) and the same reaction is expected after AVF construction when the peripheral resistance for the radial artery feeding AVF decrease. In our study we have shown that lack of an appropriate reaction after releasing a fist indicates that even after AVF construction, the arterial blood flow not increase enough for AVF function. In our prospective study, resistance index at reactive hyperemia $\geq 0.7$ is an indicator for AVF failure immediately after construction in almost $32 \%$ and internal diameter of artery $\leq 1.6 \mathrm{~mm}$ in $38 \%$ of constructed AVS fistula. Higher resistance index and lower internal diameter of artery are associated with prolonged AVF maturation time. Conclusion: Noninvasive assessment of veins by $\mathrm{Du}-$ plex sonography is very helpful in patients with inadequate clinical vein visibility. In this way we can get also more information about functional characteristics of the veins and delineated venous outflow. Routine clinical evaluation is recommended to document adequacy of arterial inflow. When indicated, especially in elderly, patients with diabetic and analgesic nephropathy, vascular renal disease, and female patients, the clinician should also order a Duplex vessels evaluation. In this groups of patients it is important to create AVF early enough and based on the results of Duplex sonography the optimal site of AVF construction should be select. The consequences of such procedure are less surgical interventions, earlier maturation of AVF, less stress for patients and at last but not at least less money for AVF surgery.

\section{4 \\ Imaging 'Work-Up' for Vascular Access in Hemodialysis}

\section{Alain Raynaud}

Hôpital Européen Georges Pompidou, Clinique Alleray-Labrouste, Paris, France

Imaging work-up concerns both the evaluation of veins before access creation and the evaluation of the access itself. Imaging of 
vein, before access creation, in patients not yet dialysed should not use iodine contrast materials because of their nephrotoxicity. Evaluation of central veins patency by MRA is simple and nearly always satisfactory. On the contrary, the spatial resolution of the MRA is insufficient for upper limb superficial veins. MRA may detect their presence but cannot evaluate their quality. Imaging of superficial veins is based on ultrasonography and a new examination the $\mathrm{CO}_{2}$ venography. $\mathrm{CO}_{2}$ venography is developing very fast. It is a breakthrough for the evaluation of superficial veins. $\mathrm{CO}_{2}$ venography requires digital subtraction angiography and a very simple equipment. When the technique of the examination is strictly respected, $\mathrm{CO}_{2}$ venography is very effective for the detection and evaluation of superficial veins. Ultrasonography appears complementary of the $\mathrm{CO}_{2}$ venography. For example, when a forearm superficial vein is detected but is irregular ultrasonography can differentiate spasm from vein wall diseases. For access evaluation, MRA and CT are improving and are currently able to satisfactorily image accesses. However they are as invasive as regular fistulographies. They require venous puncture, injection of dye (gadolinium or iodine) and heavy image processing. Moreover they do not allow interventionnal radiology in the same step. Imaging work up are rather coming from a better understanding and use of contrast materials $\left(\mathrm{CO}_{2}\right.$, diluted iodine, gadolinium). The main risk of $\mathrm{CO}_{2}$ is its reflux into vertebral artery. Thus $\mathrm{CO}_{2}$ is safe for angioaccess imaging as long as arterio-venous anastomosis opacification is avoided. When either the arteries supplying the access or the anastomosis have to be opacified, $\mathrm{CO}_{2}$ should not be used. In such cases, in patients with severe allergy to iodine, gadolinium should be used; even in case of severe renal failure, gadolinium can still be used. However, we prefer the use of highly diluted iodine contrast material $(90 \%)$. Actually the amount of iodine contrast material used for the examination is very low, less than $5 \mathrm{ml}$ for fistulography plus angioplasty. Such an amount has no effect on the renal function.

\section{Creation of Vascular Access}

Chairmen: A. Bakran, I. Barbosa

5

\section{Forearm AVF: Strategy and Microsurgical Procedures}

Pierre Bourquelota, Francesco Stortoni ${ }^{b}$

a Clinique Jouvenet, Paris, France,

b Ospedale Forlanini, Roma, Italy

We started using microsurgery in children and in adults for the creation of distal AVF in the late seventies, and preventive hemostasis in the early nineties [1]. In children our results for AVF microsurgery under $10 \mathrm{~kg}$ were published in 1981 [2]. In 1990 [3] we reported 380 children receiving microsurgery for AVF: the immediate patency rate was $96 \%$ and the 24 -month patency rate was $85 \%$ in distal radial-cephalic AVF. The benefit of microsurgery for children was emphasised in 1984 by Yazbeck [4], in 1993 by Sanabia [5] and in 1998 by Bagolan [6]. In adults also (Lim, Margic, Luchian) microsurgery in angioaccess gaining is a great benefit for the patient and a valuable teaching instrument too. Conservative use of the venous system is a must in every patient doomed to hemodialysis. When possible, the dorsal veins of the hand must be used for blood sampling. Absolute respect of the non-dominant arm is necessary. Subclavian central venous catheters with high risk of proximal vein stenosis are prohibited. Careful preoperative clinical examination and Doppler examination assess the condition of the vein; a venous angiography (iodine or $\mathrm{CO}_{2}$ ) is necessary if there is any doubt, particularly when central venous catheter has been inserted previously. Prophylactic broad-spectrum perioperative antibiotic is prescribed. The rules for suturing are: forceps must never grasp the intima, the adventitia is incised, not resected, high pressure clamps must be avoided, the thinnest possible needles are to be used. Preventive hemostasis, using a pneumatic tourniquet, makes extensive arterial dissection for clamping unnecessary: arterial spasm and ischemia are avoided. When hemostasis is incomplete, micro-clamps are placed on the artery after minimal dissection.

\section{Various AVF}

(1) Radial to Cephalic AVF: The cutaneous incision is longitudinal, half way between the cephalic vein and the radial artery in the wrist. The nerves must be carefully preserved. The vein is freed by a wide dissection, a ligature being placed on the collateral branches: this avoids the use of an electric scalpel that could damage the vascular trunk itself. The diameter of the vein is measured. The vein is irrigated frequently in order to prevent drying and it is only handled by its adventitia. After section of the vein above a distal ligature, posterior incision of approximately $10 \mathrm{~mm}$ in length is made in the proximal vein. The anterior wall of the artery is exposed, without dissecting the arterial trunk. A longitudinal arteriotomy is commenced with a disposable ophthalmologic scalpel, taking great care not to damage the posterior wall. This is completed with scissors. If a few drops of blood appear they are rinsed with heparin saline and the blood is dried with small sponges. The anastomosis is made with four running sutures, by suturing first the proximal angle. It is preferable to keep the running suture untightened at the beginning so as to leave the lumen of the artery open. Frequent use of the zoom facilitates this very precise part of the operation; of course the needle must be repositioned in the needle holder for each passage through a vessel. Both proximal running sutures (posterior and anterior) are stopped at the middle of the arteriotomy. The rest of the anastomosis is made in starting from the distal angle. The tourniquet is then released. If there is a leak, an additional suture is added. Patency is confirmed by Doppler ultrasound. The murmur can be absent during the first postoperative hours particularly in small children. It is very important to ascertain that the vein is not stenosed at the upper limit of the dissection.

Variations: Two Running Sutures Anastomosis: The proximal suture is made first, starting at the middle of the posterior wall of the arteriotomy, going up to the proximal angle, and down to the middle of the anterior wall. The distal suture is made afterward. This technique gives an excellent vision on the arterial lumen. Transposition: It is sometimes necessary to surgically transpose subcutaneously the vein two months later in small children with thick adipose tissue or in obese patients. An interval of three weeks must be respected before puncturing. 
(2) Ulnar to Basilic AVF: A sufficient length of the vein, which is situated quite far back from the artery, must be freed. It can be a help to put the forearm in flexion at the elbow during dissection. The artery is approached by passing behind the flexor carpi ulnaris, which can be transected. The interval before puncture is longer than with a radial to cephalic AVF.

\section{Strategy}

All patients must have a microsurgical radial to cephalic or ulnar to basilic distal AVF, if possible, created one month before use (2 to 3 months in children). In children under $10 \mathrm{~kg}$ the delay is longer. Arterio-venous grafts, still recommended by a few authors, must be avoided at all costs in children [7] in view of the rapidity with which they become complicated by stenosis of the venous anastomosis. In adults, graft is a second choice after distal and proximal AVF. In case of emergency and if peritoneal dialysis is impossible, a central venous catheter must be inserted. A subclavian catheter is to be avoided because of the risk of interference with the venous drainage of the limb with dramatic consequences for the future. An internal jugular vein catheter is preferable. Microsurgery has greatly improved and simplified the creation of distal AVF. In adults long term patency of distal AVF is observed frequently, up to 25 years. In children microsurgery has virtually eliminated the use of long term venous catheters and arterio-venous grafts.

\section{References}

1 Bourquelot P: Preventive haemostasis with an inflatable tourniquet for microsurgical distal arteriovenous fistulas for haemodialysis. Microsurgery 14:462-463, 1993.

2 Bourquelot P, Wolfeler L, Lamy L: Microsurgery for haemodialysis distal arteriovenous fistulae in children weighing less than $10 \mathrm{~kg}$. Proc Eur Dial Transplant Assoc 18:537-541, 1981.

3 Bourquelot P, Cussenot O, Corbi P, Pillion G, Gagnadoux MF, Bensman A, Loirat C, Broyer M: Microsurgical creation and follow-up of arteriovenous fistulae for chronic haemodialysis in children. Pediatr Nephrol 4:156-159, 1990.

4 Yazbeck S, O'Regan S: Microsurgery for Brescia-Cimino fistula construction in pediatric patients. Nephron 38:209-212, 1984.

5 Sanabia J, Polo JR, Morales MD, Canals MJ, Polo J, Serantes A: Microsurgery in gaining paediatric vascular access for haemodialysis. Microsurgery 14:276-279, 1993.

6 Bagolan P, Spagnoli A, Ciprandi G, Picca S, Leozappa G, Nahom A, Trucchi A, Rizzoni G, Fabbrini G: A ten-year experience of Brescia-Cimino arteriovenous fistula in children: technical evolution and refinements. J Vasc Surg 27:640-644, 1998.

7 Bourquelot P, Gagnadoux MF: Vascular access for hemodialysis in children. Pediatr Nephrol 11:659-660, 1997.

\section{6 \\ Upper Arm Arterovenous Fistulas Using Native Vessels}

\section{Berardinelli}

Division of Vascular Surgery and Kidney Transplantation, Policlino Hospital IRCCS, Milan, Italy

Whenever the forearm vessels are unsuitable for constructing a radiocephalic fistula, a proximal elbow fistula (PEF), using the patient's native vessels ought to be always preferred to the implantation of a prosthetic graft, that presents higher rates of complications and lower long term patency. In children who present smaller arterial size, or in older/diabetic patients with atherosclerotic/calcified arteries, the PEF can be downright considered as a first choice procedure. In these cases, the proximal anastomosis between the brachial artery and an antecubital vein allows the blood to flow distally in a retrograde fashion, thus widening the forearm veins, which could be totally destroyed by multiple failed access procedures. The suitability of the brachial artery at the elbow can be easily assessed by simple palpation, although anatomical malformations can be observed at this site with an incidence of $0.14 \%$. It is mandatory to process with a venogram enlarged to the ipsilateral axillary and subclavian vein to detect occult central venous obstruction in patients, who had prior temporary hemoaccess procedures. Local anesthesia by infiltration of $1 \%$ lignocaine is always preferred with minimal patient discomfort, even in willing children under 7 years. A transverse incision of 2 or $3 \mathrm{~cm}$, just below the elbow crease, is sufficient to expose the antecubital veins. The brachial artery is exposed through the same cut, severing the fibrous end of the bicipital aponeurosis. The median cephalic or median basilic vein can be used for a side-to-side anastomosis with the brachial artery; whenever these veins are not adequate, the 'perforating' vein can be used for an end (vein)-to-side (artery) anastomosis. Another technical combination, particularly useful when the superficial veins at this site are fibrous or anyhow damaged, consists in a side-to-side anastomosis with the 'comitans' vein. Other combinations are however possible, mobilizing the basilic or the cephalic vein. Decrease in perfusion of the distal limb, up to a 'steal syndrome', can be easily observed in the PEF, if the length of the arterial mouth is not kept shorter than $80 \%$ of the arterial diameter. We usually do not attempt the salvage of a PEF that is failing after a long term patency: in this case, as well as in the few cases of non optimal maturation of the venous bed, a prosthetic graft can be easily placed between the same vessels of the PEF, just above. In conclusion, the PEF, constructed adopting some cautions, shows excellent early and late patency rates, proving too to be the best choice as first vascular access procedure for the smallest and oldest uremic population.

7

\section{PTFE Graft}

J osé R. Polo

Vascular Access Unit, Hospital General Universitario

Gregorio Marañon, Madrid, Spain

Indications: A graft for dialysis should be placed only when a forearm or upper arm autologous fistula cannot be performed due to lack of adequate superficial veins. Due to the high incidence of graft complications, peritoneal dialysis should be considered before a graft is constructed. Alternative permanent dacron cuff catheters should be considered in patients with a short life expectancy in which an autologous access cannot be performed. Anatomical position of grafts: Most grafts are placed in the forearm either in a straight shape between the radial artery and a elbow vein or in a curve shape (the so called loop graft) between the brachial artery and an elbow crease (Palder S et al, Ann Surg 1985;202:35-39). Whenever an elbow crease fistula is preferred when an upper arm vein seems to be adequate most of grafts are placed at the upper arm between the brachial artery and a large vein in the lower part of the axilla (Polo et al, 
Artif Organs 1995;19:1181-1184). Other less frequent positions are: brachioaxillary (Polo JR et al, Am J Kidney Dis 1990;16:465-468); axillo-axillary (McCann RL, J Vasc Surg 1996;24:457-462); femorofemoral (Korzets A et al, Nephrol Dial Transplant 1998;13:12151220); axillofemoral (Rueckerman et al, ANNA J 1991;18:567-571). In cases of caval obstruction a graft to the right atrium has been performed (El-Sabrout RA, Duncan JM et al, J Vasc Surg 1999;29:472478). On the other hand arterio-arterial grafts in several positions have been used in cases of obstruction of superior and inferior vena cava (Scholtz H et al, in Henry ML Proceedings of Vascular Access for Hemodialysis VI, Miami, Precept Press, 1999, pp. 255-262). Technical approach: My personal choice is to perform all upper arm arteriovenous grafts, included brachiojugular prosthesis, under local anesthesia and in an ambulatory surgical setting (Polo et al, Amb Surg 1997;5:149-151). After dissection of the artery and the vein a tunneler is introduced under the skin and the graft is immediately in the subcutaneous space avoiding a long exposure of the prosthesis in the operating field. The graft vein anastomosis is first performed and a clamp is placed in the graft close to the vein. The venous micro-bulldogs are released and the graft vein anastomosis is checked under a low pressure flow. Afterward, the arterial graft anastomosis is performed and, before the suture is tied, the graft clamp placed in the venous site is released allowing the blood fill the graft. The arterial anastomosis is then tied and the arterial clamps removed. In this way, blood remain flowless in the graft for a few second and heparin is not needed. Long term results: I will present my experience with 716 grafts performed between 1981 and 1997. In this period the following accesses were performed in our unit: 1,259 radiocephalic arteriovenous fistulae (AVF) (67\%), 468 upper arm AVF (18\%), 82 dacron cuff catheters (3\%) and 716 PTFE grafts (28\%). Anatomical position of grafts were as follows: upper-arm (6-8 $\mathrm{mm}) 324$ (45\%); upper-arm (6 mm) 184 (26\%); straight forearm (6 $\mathrm{mm}) 96(13 \%)$; curve forearm $(6 \mathrm{~mm}) 49(7 \%)$; brachiojugular $(6$ and 6-8 mm) 51 (7\%); femoro-fermoral (6-8 mm) 12 (2\%). Overall complication rate was of 0,49 episodes per graft-year of follow-up. Complication rate (episodes per graft-year) was significantly lower in 6-8 mm upper arm grafts $(0.33)(\mathrm{p}<0.001)$, comparing with the rest of grafts $(6 \mathrm{~mm}$ upper-arm $=0.57 ; 6 \mathrm{~mm}$ straight forearm $=0.62$; brachiojugular $=0.72 ; 6 \mathrm{~mm}$ curve forearm $=0.74)$. Overall primary patency was 70,34 and $12 \%$ at 1,5 and 10 years. Overall secondary patency (after successfull rescue surgery for complications) was: 86 , 66 and $39 \%$ at 1,5 and 10 years. Best patency rates were observed in 6-8 mm upper-arm grafts: primary, $92,52,24 \%$ at $1,5,10$ years; secondary, 96, 79 and 54\% at 1, 5, 10 years (Polo et al, Br J Surg 85:(suppl 2):4). To get a good secondary patency, a close program of surveillance (better with access flow measurements), and early treatment of thrombosis and stenosis is mandatory.

\section{8 \\ Biological Graft as Vascular Access for Hemodialysis \\ Massimo Morosetti \\ Divisione di Nefrologia e Dialisi, Ostia-Roma, Italy}

Vascular access dysfunction is the most important cause of morbidity and hospitalisation in the hemodialysis (HD) population in USA at a cost of 1 billion of dollars per year. In spite of current recommendation of placement of a native artero-venous fistula, PTFE grafts continue to be the functioning vascular access in the majority of patients on HD in the USA. Also in Europe HD access achieved through a prosthetic vascular graft has become more popular, especially in diabetic and older patients and those who had several unsuccessful surgical accesses. Venous neointimal hyperplasia characterized by stenosis and subsequent thrombosis accounts for the overwhelming majority of pathology resulting in PTFE graft failure. Was reported that graft failure is higher in patients with an initial anastomosing vein diameter smaller than $4 \mathrm{~mm}$ than in those with a

\section{Biological Prosthesis}

\begin{tabular}{ll}
\hline Advantages & Disadvantages \\
\hline Handy & Biodegradation \\
Elasticity & Costs \\
Flexibility & Immunogenicity \\
Compliance & \\
Patency & \\
Hemocompatibility & \\
Resistance to infections & \\
Resistance to sticks & \\
No seroma & \\
No thrombogenesis &
\end{tabular}
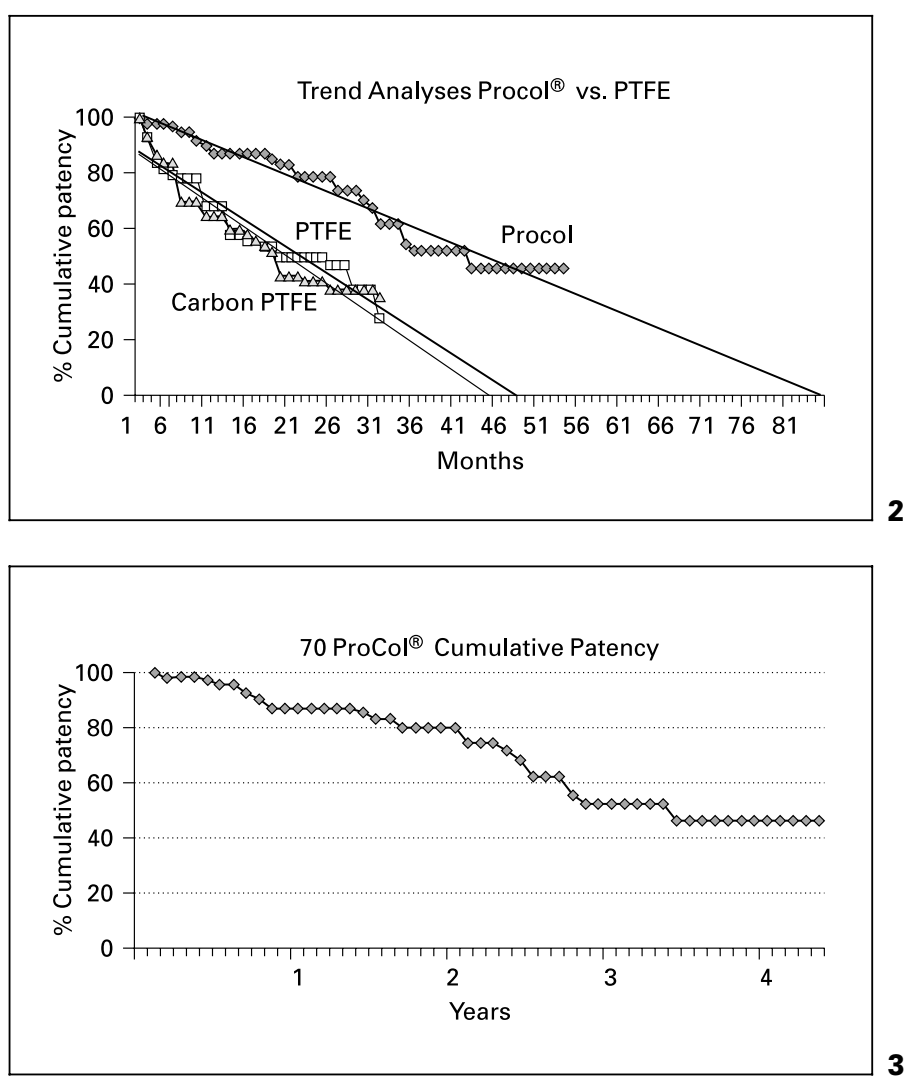
vein diameter of $4 \mathrm{~mm}$ or longer. Seroma is one other frequent complication of PTFE graft. It is a collection of clear, proteinaceous and sterile fluid, confined within a non-secretory fibrous pseudomembrane surrounding a PTFE graft. Was also been reported that infection of PTFE graft accounts for a significant number of hospitalisation and uses a large amount of healthcare resources. An ideal AV prosthesis for HD should have good biocompatibility, flexibility, compliance, long-term patency, resistance to infections and to multiple sticks and, not least, easy to use from the surgical point of view. Biological prosthesis has been used as peripheral vascular graft for
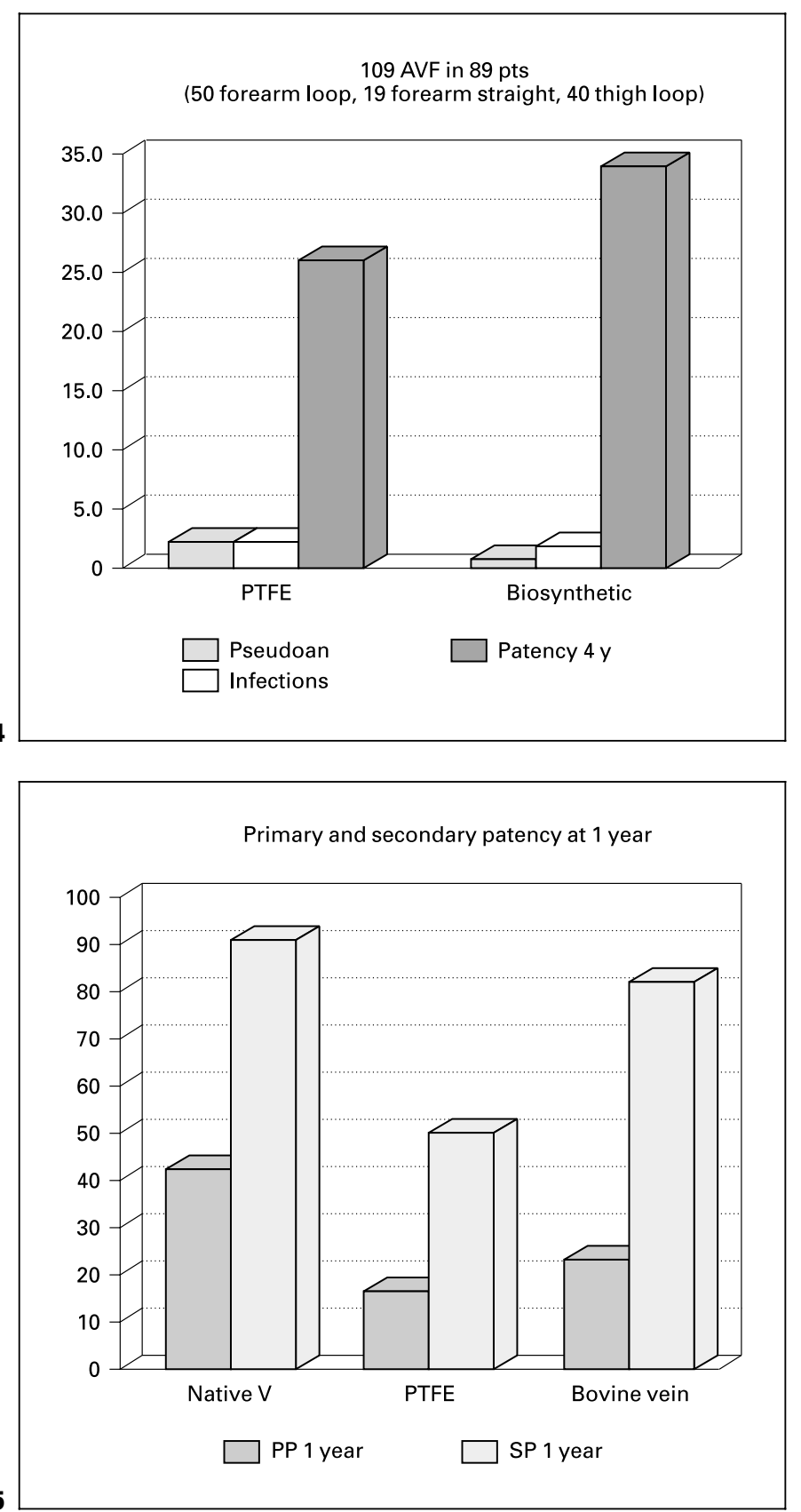

4th Basic Multidisciplinary Hemodialysis Access Course long time and more recently as artero-venous bridge for HD. At the moment biological graft mostly used on HD patients are mesenteric bovine vein (Procol) and ovine collagen over a polyester net (Omniflow II-biosynthetic graft). Advantages and disadvantages of the biological graft are listed in figure 1. Biosynthetic prosthesis show biocompatibility of biological grafts and patency of synthetic grafts; indeed collagen account for the biocompatibility and hemocompatibility and polyester leads to a long-term resistance. Furthermore it has been reported that glutaraldehyde treatment improves the elasticity, masks the antigenicity and sterilizes the graft, combining the resistance of synthetic graft and biocompatibility of biological graft. In figures 2, 3 is reported cumulative patency of the biological graft and trend analyses versus synthetic ones. The primary and secondary patency is better in biological graft when compared to synthetic one (fig. 4) and the incidence of complications is lower (fig. 5). In conclusion the biological prosthesis when placed as secondary vascular access for hemodialysis show better long-term patency, more ease to use and lower incidence of complications.

\section{9 \\ Vascular Access in Children}

\section{Giorgio Fabbrini}

Department of Pediatric Surgery, Bambino Gesù Hospital, Research Institute, Rome, Italy

Introduction: Vascular access for chronic hemodialysis is a surgical challenge in pediatric patients. External shunts restrict the child's physical activity, do not last for a long time and are responsible for higher complication rates. As a consequence arteriovenous fistula (AVF) is probably the first choice because suitable vessels for distal angioaccess are available in nearly all these patients despite their age and weight. Materials and Methods: From January 1985 to December 2000, 170 Brescia-Cimino AVFs were performed in 122 children. The main group included 148 Brescia-Cimino AVFs (BC-AVF) in 116 patients with an average age of 9.5 years (range 5 months to 18 years). The average weight was $33.5 \mathrm{~kg}$, ranging from 6.5 to $65 \mathrm{~kg}$. $13 \%$ of BC-AVFs was less than 5 years of age and $15 \%$ weighed less than $15 \mathrm{~kg}$. Results: Chronic renal failure was caused by a nephropathy in 74 cases and 42 had a uropathy. In all cases a flebography was performed before the microsurgical treatment. Since 1994 an inflatable tourniquet has been placed in the selected upper arm because an optimal exanguination of the operating field. The primary patency rate was obtained in 104 out of 116 children $(90 \%$ of whom weighed less than $15 \mathrm{~kg}$ and $89 \%$ more than $15 \mathrm{~kg}$.) $50 \%$ of BC-AVFs had either immediate or late complications. Thrombosis was the most frequent complication that we observed. In comparison with $4 \%$ of late complications, $13 \%$ of early complications caused the loss of the access. Out of 102 BC-AVFs, $63 \%$ with a 4 year follow-up are still patent. Out of 79 BC-AVFs with a 5 year follow-up, $59.5 \%$ are still patent. Conclusion: We emphasise that: (1) Microsurgery is essential to create BC-AVFs in children with good results in children as well as in adult patient. (2) The result improved after the adoption of an upper-arm exanguination and ischemia. (3) BC-AVF remains the more satisfactory method for providing haemodialysis access in children.

Preprint Blood Purif, Vol. 20, 2002 


\section{Vascular Access Complications}

Chairmen: M. Lazarides, J. Polo

\section{0 \\ Factors Predicting or Influencing Early Failure of AV Fistulae}

\section{A. Bakran \\ University of Liverpool, The Royal Liverpool University \\ Hospital, Liverpool, UK}

It is suggested that up to $30 \%$ of radiocephalic AV fistulae fail, either due to early thrombosis or because the fistula vein does not dilate to allow haemodialysis [1]. This failure rate may be due to diseases of the radial artery itself, inadequacy of the cephalic vein, to poor surgical technique as well other host factors. The latter can only be addressed by better training and meticulous attention to detail in the performance of surgery. However, there is now sufficient evidence that preoperative duplex scanning can elucidate the morphology of artery and vein before surgery, thereby allowing problems to be picked up before surgery. Research in Liverpool has shown the duplex scanning is better at detecting diseases of vessels than clinical examination alone [2]. Disappointingly, intraoperative flow measurements have not been predictive of fistula success but duplex scanning during the follow up period has. The velocity of flow measurements at 24 hours post AV fistula creation has been shown to distinguish between those fistulae which will go on to be successful and allow haemodialysis (mean velocity of flow 0.53 metres per second) compared to those more likely to fail (mean velocity of flow

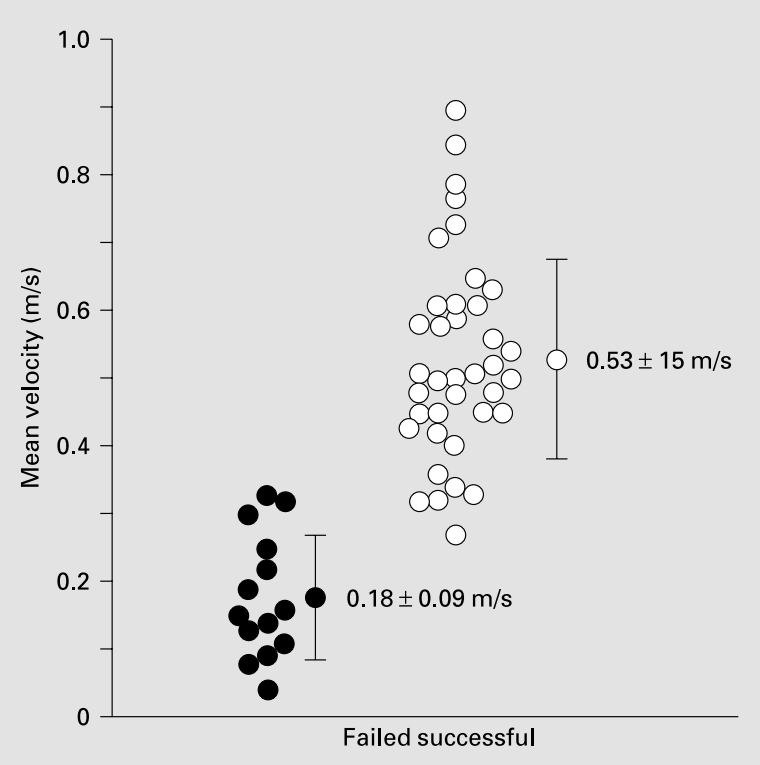

1
$<0.2$ metres per second). Furthermore, duplex scanning of AV fistula over the subsequent few weeks to assess fistula blood flow and vein diameter has been shown to separate the successful and failing fistula pattern. Therefore, measurements of velocity of flow at 24 hours and fistula flow at two/three weeks post-operative should provide good predictions of success or failure of AV fistulae. This allows surgeons to either revise the unsuccessful fistula, or to create another AV fistula at a different site. No longer need a surgeon prevaricate in his decision making when reviewing patients with radiocephalic AV fistula problems following surgery.

\section{References}

1 Palder SV, Kirkman RL, Whittlemore AD, Hakim RM, Lazarus TM, Tilney NL: Vascular access for haemodialysis: Patency rates and results of revision. Ann Surg 1985;202:235-239.

2 Wong V, Ward R, Taylor J, Selvakumar S, How TV, Bakran A: Factors associated with early failure of arteriovenous fistulae for haemodialysis access. Eur J Vasc Endovasc Surg 1996;12:207-213.
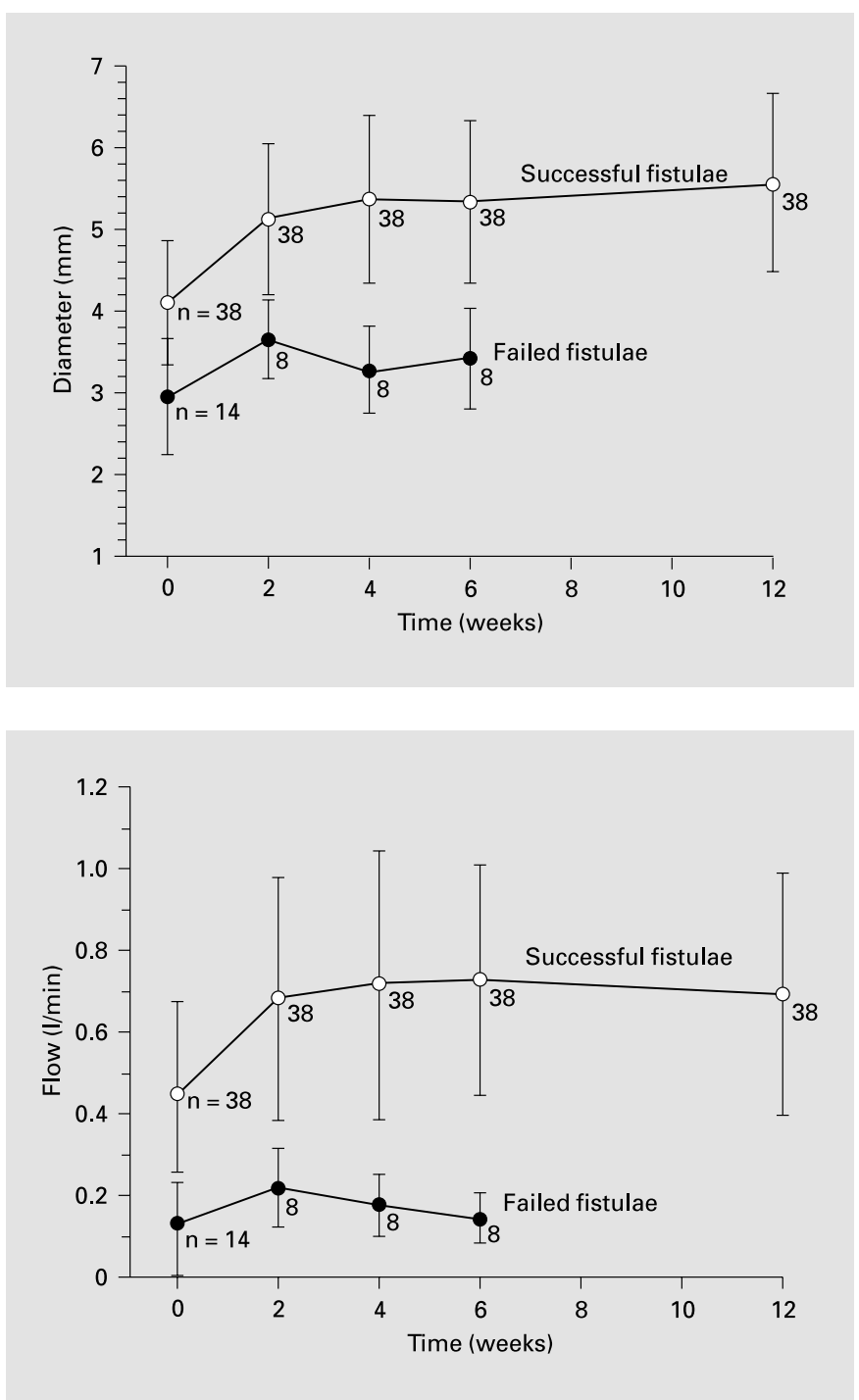


\section{1 \\ Vascular Access and Intimal Hyperplasia}

\section{J .H.M. Tordoir}

Department of Surgery, University Hospital Maastricht, The Netherlands

The most prominent complication of graft AVF failure is uncontrolled intimal thickening by proliferation of smooth muscle cells, i.e. intimal hyperplasia, leading to progressive stenosis and subsequently thrombosis.

\section{Factors Influencing Intimal Hyperplasia}

Blood Flow and Intimal Hyperplasia: Intimal hyperplasia often occurs at the graft-vein anastomosis and in the efferent vein. Presumed to be of great importance in the development and progress of intimal hyperplasia is a certain hemodynamic factor which involves the interaction between the vessel wall and the bloodstream, namely wall shear stress (WSS). WSS is the frictional force exerted by the circulating blood column on the endothelial monolayer covering the inner vessel wall. WSS is believed to play a significant role in the physiological adaptation of the vascular wall, in that it induces artery dilatation with increasing volume flow and vice versa. Acute changes in blood flow induce the release of vasoactive molecules by the endothelium, whereas chronic changes induce vascular remodeling. WSS is associated with the development of intimal hyperplasia. There is no conclusive data indicating whether high or low WSS is related to the development of intimal thickening. The endothelial cell plays a pivotal role in this process. Mechanisms are mechanotransduction processes that lead to biophysical, biochemical and gene regulatory responses of endothelial cells to hemodynamic shear stresses. These mechanisms include endothelial-mediated alterations in coagulation (prostacyclin, NO, protein $\mathrm{C}$ and $\mathrm{S}$ ), leukocyte and monocyte migration, proliferation of smooth muscle cells (PDGF, angiotensin II, NO, TGF- $\beta$ ), and signal transduction.

Peri-Anastomotic Mismatch in Elastic Properties: In addition to WSS, peri-anastomotic compliance mismatch, i.e. mismatch in elastic properties, seems to be an important factor in the development of intimal hyperplasia. A mismatch in elastic properties may affect intimal hyperplasia in 3 ways: (1) Mismatch leads to a point of excessive mechanical stretching, which may result in vessel wall injury and initiate a vascular response resulting in intimal thickening. (2) Cyclic stretching has a positive influence on replication of VSMC and production of extracellular matrix (ECM). In vitro studies have demonstrated that VSMC start to produce ECM and to replicate when they are subjected to high levels of distension. This phenomenon is more pronounced in VSMC derived from veins. (3) Compliance may influence flow characteristics. Flow studies have demonstrated that a sudden increase in compliance is associated with enhanced particle residence time, flow stasis and separation, suggesting low shear conditions.

In conclusion, it might be proposed that WSS, endothelial cell functioning and compliance mismatch or their possible relationship are of importance in the development of intimal hyperplasia and stenoses.

\section{2}

\section{Monitoring of Vascular Access and Screening of Stenosis}

\section{F. Cavatorta a, A. Zollo a, S. Galli a, R. Ervo a, P. Dionisio ${ }^{b}$}

a Department of Nephrology, General Hospital, Imperia,

bDepartment of Nephrology, General Hospital, Biella, Italy

Malfunction of permanent vascular access, which includes native vein fistulae (AVF) and polytetrafluoroethylene (PTFE) grafts, remains a cause of frequent and costly morbidity in patients receiving chronic hemodialysis (CHD). Failure ensues in more than $80 \%$ of cases because a thrombotic episode cannot be resolved. A careful approach will help to sustain life and quality of life longer than if access systems are constructed and monitored carelessly. The Dialysis Outcome Quality Initiative recommends a regular program of access screening as part of an overall continuous quality improvement program to improve access management. The methods for identifying access sites at increased risk for thrombosis include physical examination, dynamic venous dialysis pressures (DVDP), static venous pressures (SVP), recirculation, Doppler ultrasound, in-line blood flow measurement. DVDP is a simple and inexpensive method of measuring intra-access pressure in which venous drip chamber pressure is recorded within the first $2-5$ minutes into each dialysis. Three consecutive values exceeding a pre-established threshold $(125-150 \mathrm{~mm} \mathrm{Hg})$ trigger referral for angiography. Trend analysis is much more important than any single value. Measurement of static intra-access pressure (SVP), at a dialyzer blood flow of $0 \mathrm{ml} / \mathrm{min}$, eliminates flow effects and the effects of partial occlusion of the needle orifice. Under normal conditions the drip chamber records a pressure less than the pressure in the access. This difference correlates with the height difference between the access and the top of the blood in the drip chamber $(\Delta \mathrm{PH})$. SVP is equal to the drip chamber pressure added to $\triangle \mathrm{PH}$. SVP can now be performed without separate devices. Recirculation occurs when access flow rate falls below the pump flow rate. In general, even prosthetic grafts at high risk for thrombosis, in contrast with AVF, have flow rates that exceed pump flow rates currently used for dialysis. Recent evidence indicates that test of recirculation may play some role in screening native fistulae, but have been deemed not useful in detection of early access stenosis in PTFE.

Direct intra-access measures of blood flow are currently the most accurate method for detecting access stenosis. All methods of measuring direct access flow rely on the Fick principle. To measure blood flow, specific apparatus (Transonic system) is necessary, which is based on the ultrasound dilution technique and the inversion of the inlet and outlet dialyser blood lines. Data obtained by ultrasound dilution suggest that access flow of less than $600 \mathrm{ml} / \mathrm{min}$ in PTFE and decrements in access flow of $>15 \%$, if the flow is less than 1,000 $\mathrm{ml} / \mathrm{min}$, is likely to predict access thrombosis and hemodynamically significant stenosis. Next to the Transonic system, blood flows can also be measured by Doppler ultrasonography. Colorflow Doppler has a confounding variable, however, flow measurements are operator- and machine-dependent and have significant variability. Although the focus is frequently on technological methods for access evaluation and monitoring, it is important to perform adequate clinical evaluation for access stenosis. It is also very useful prior to the placement of a peripheral vascular access. Findings suggesting access disfunction are recurrent clotting (more than one episode per month), difficult needle placement, difficulty with attaining hemo- 
stasis (within 20 min of needle withdrawal) and persistently swollen arm with multiple, dilated veins and collateral veins on the upper arm and chest. Significant venous stenosis causes hemodynamic changes in AVF and in PTFE. These changes result in abnormalities that can be detected by physical examination. A properly functioning vascular access has a soft, easily compressible pulse with a continuous palpable thrill only at the arterial anastomosis. It has a lowpitched bruit, which is continuous with both systolic and diastolic components. With severe stenosis the bruit is high pitched, discontinuous and only systolic. Another sign of low flow is a discontinuous, water-hammer type of pulse. A localized increase in pitch suggests stenosis. Despite the limitations of physical examination, it should be performed at monthly intervals, particularly in programs without other monitoring capabilities. In fact, it was demonstrated a significant drop in the access thrombosis rate after institution of a surveillance program that included physical examination, DVDP, and recirculation. Abnormal physical examination findings were the most common sole indicators of graft dysfunction. crossed an externally ringed graft is needed to avoid kinking. (3) Junctional stenosis. In brachiocephalic/basilic AVFs, junctional stenoses occur frequently. PTA is the first option for treatment of basilic vein junctional stenosis and surgical patch plasty may be needed after failed PTA. Thrombosis in AVFs with transposed basilic veins usually leads to clot propagation into the entire vein. An attempt to successful thrombectomy must be performed within several hours after clotting. A primary surgical approach is advised of stenoses at the site of the cephalic vein entering into the subclavian vein, because of the risk of rupture during PTA. A patch plasty may be a difficult operation and alternative procedures like transposition of the proximal cephalic vein to the axillary vein is recommended. In conclusion, a surgical revision of stenoses/thrombosed AVFs can be performed with good success rates by patch plasty, graft interposition or vein transposition \pm thrombectomy.

\section{4}

\section{No abstract received}

\section{Treatment of Stenosis and Thrombosis}

\author{
Chairmen: V. Mickley, L.T. Rodrigues
}

\author{
13

\section{Surgical Approach/AVF} \\ J.H.M. Tordoir \\ Department of Surgery, University Hospital Maastricht, \\ The Netherlands
}

The most common cause for low flow and/or thrombosis of native AVFs is a stenotic lesion or vessel segment, induced by intimal hyperplasia. The typical location of stenoses in radiocephalic AVFs is the arteriovenous anastomosis ( $3 / 4$ of cases) and outflow vein ( $1 / 4$ of cases). In brachiobasilic/cephalic AVFs the stenosis is usually located at the junction with the axilary or subclavian vein. Surgical approach depends on the location of stenosis/thrombosis: (1) Anastomotic region. Intimal hyperplasia is best managed by one of three methods: a) widening of the lumen with a patch angioplasty \pm thrombectomy; b) interposition of a short segment of prosthetic graft from the artery to a suitable (open) outflow vein; c) new proximal anastomosis of the cephalic vein to the radial artery. In most cases the stenotic lesion is located in the proximal cephalic vein just adjacent to the anastomosis. If the proximal radial artery is stenosed, it is easier to perform a new proximal anastomosis or to transpose the radial artery to the cephalic vein. (2) Venous outflow stenosis. The primary choice of short-segment outflow stenosis $(<2 \mathrm{~cm})$ is percutaneous transluminal angioplasty (PTA). Long-segment stenoses are treated by graft interposition or basilic vein transposition in the forearm. This method has the advantage that only one anastomosis is needed and the risk on intimal hyperplasia usually occurring at the graft-vein anastomosis is avoided. When the basilic vein is not suitable, because of small diameter, a graft is interposed in the forearm. When the elbow must be

\section{5 \\ Endovascular Treatment of Stenosis: Dilatation}

L. de Medici, S. Pieri

A.O.S. Camillo Forlanini, Rome, Italy

Because of paucity of acceptable alternatives, prolonging the patency of permanent dialysis access is crucial to maintaining life. Furthermore, major attention is focused today on flow and capacity of vascular access. Percutaneous transluminal angioplasty (PTA) has changed the care of the permanent dialysis access by providing a relatively noninvasive repeated method to treat the stenoses that inevitably develop, while preserving the veins during a continuous followup of the vascular access. Attention to details and determining procedure endpoints are essential for good outcomes. The steps of procedure are the choise of percutaneous puncure site, the balloon, the inflation time, drugs during and after the procedure, the immediate endpoints of ballon angioplasty. Determining the failures of the dilatation (unable to efface waist on the ballon - elastic recoil - venous rupture) and how to solve them is also important. Nevertheless, there are significant limitations to balloon angioplasty in this patient population. First, chronic catheter related central vein lesions, expecially occlusions, are less successfully treated with balloon angioplasty than peripheral venous lesions. Second, depending on lesion location, elastic recoil after attempted balloon angioplasty is known to occur in $37-64 \%$ of cases, and results in immediate treatment failure. Finally, the 6 month cumulative primary patency rate after balloon angioplasty is $38-63 \%$ for screened patient population, and only $18-39 \%$ after thrombolysis/thrombectomy. Percutaneous transluminal angioplasty is the first step in dialysis access intervention, but these limitations have spurred on a search for more effective and durable adjuncts to balloon dilatations. 


\section{6 \\ Endovascular Treatment of Stenosis - Stent Placement}

P. Haage, D. Vorwerk, R.W. Günther

Department of Diagnostic Radiology, University of

Technology, Aachen, Germany

More than $85 \%$ of patients with end-stage renal disease (ESRD) are treated by hemodialysis at some point in their life. As reported by the United States Renal Data System (USRDS) hemodialysis access failure is the most frequent cause of hospitalization among ESRD patients and accounts for the largest number of hospital days in some centers. This implicates the enormous importance of an adequate maintenance of the vascular access. Management of venous stenosis accounts for most of the vascular access related procedures the interventional radiologist is confronted with in daily clinical practice. Predisposing factors and signs indicative of stenosis include: previous thrombosis, decreasing access flow, elevated venous dialysis pressure, abnormal urea parameters and a decrease in measurement of dialysis dose. Percutaneous transluminal angioplasty (PTA) has been proven to be an effective, safe and comparatively inexpensive procedure in venous stenoses with impressive technical success rates. Advantages over a surgical approach range from the ability to perform the intervention on an outpatient basis and perform dialysis immediately post procedure to repeatedly being able to treat the same lesion without sacrificing valuable vein. Stent placement should be reserved to those cases not appropriately treatable by PTA alone, such as highly rigid stenoses and stenoses with high recoil properties. Vessel dissection after balloon angioplasty is another indication for shunt preservation through stenting. In those cases stents may aid in improving results. In centers where surgery is the treatment of choice, percutaneous treatment should be considered when residual access sites are limited or the lesion is not surgically accessible. The idea of also preventing restenosis has shown that stents do not decrease the likelihood of recurrence. Thus primary stent placement, which may be considered in central venous obstructions, is not recommended. Endoprostheses should not be placed in puncture areas. For forearm and upper-arm implants, the stent should be flexible yet strong enough to endure external pressure due to its superficial location. Moreover, recoil to its initial shape after release of compression is required. A self-expanding stent such as the Wallstent is a suitable device with a strong radial force nevertheless avoiding adjacent nerve damages and ensuring safe fixation. The size of the delivery system should be moderate and stent length should be kept as short as possible, covering the entire lesion with a slight overlap. To avoid stent infection, deployment should be delayed, if there are signs of active infection in the patient. By restricting stent placement to selected cases with the appropriate indication, it can be a valuable and helpful support for the patient and his or her physician.

\section{7 \\ Percutaneous Declotting of Arteriovenous Prosthetic Grafts for Dialysis}

\author{
Luc Turmel-Rodrigues \\ Clinique St-Gatien, Tours, France
}

Acute graft thrombosis should be avoided and warrants surveillance programs for detection and correction of stenoses which underlie more than $85 \%$ of thromboses. Trerotola published a controversial article in 1994 which was a milestone in the history of hemodialysis access declotting because it explained everything before and after. He simply pushed the clots into the lungs. Trerotola justified this technique by the fact that the average volume of thrombus in a clotted graft is only $3.2 \mathrm{ml}$, which explains why this modest iatrogenic pulmonary embolism is asymptomatic in the vast majority of cases. This key article explains why so many declotting methods have been reported and are still likely to be reported: everybody can claim to remove clots with more or less efficient devices or drugs since residual clots can eventually simply be pushed into the lungs. Unfortunately several articles have since related casualties due to thrombus migration during graft declotting. The percutaneous declotting techniques published to date can be divided into pharmacomechanical and purely mechanical methods. Some are cheap, others are expensive (device cost $>500$ Euros). Among pharmacomechanical methods, thrombolytics at low or high dose are infused locally through regular needles or specific catheters for some minutes or hours before detachment or crushing of residual thrombi with a balloon: these are the 'pulse-spray' method of Bookstein, the 'lyse and wait' method of Cynamon, the infusion technique of Goodwin, and the Craggbrush. Purely mechanical methods include the balloon-based methods of Trerotola and Sharaffuddin, manual catheterdirected thromboaspiration, the pulse-spray with saline of Beathard, the Gelbfish device, the rotating pigtail of Schmitz-Rode and all kinds of declotting machines. Among these machines, some have a direct mechanical action (Arrow-Trerotola PTD ${ }^{\circledR}$, Craggbrush $^{\circledR}$ ) but the majority work on the Venturi effect or on the creation of a vortex: Hydrolyser $^{\circledR}$, Amplatz-Thrombectomy Device ${ }^{\circledR}$, Angiojet $^{\circledR}$, Oasis ${ }^{\circledR}$ and many others likely to come. There is a consensus in the American literature to consider that the outcome of a declotted graft depends on how the underlying stenosis is treated and not on how the thrombus is removed, which is probably true as long as the whole thrombus is actually removed. Does this mean that all methods are equivalent? Are the wide differences in success rates and in the rates of early rethrombosis only explained by different populations? Long-term primary patency rates are relatively poor in all reports, ranging from $8-26 \%$ at 1 year. However secondary patency rates of $75 \%$ have been reported. DOQI guideline 21 mentions that an $85 \%$ initial success rate must be achieved for both surgery and radiology and most of the recent studies reached this goal. The $40 \%$ rate suggested at 1 year for surgery was achieved but none of the 3 most recent studies since they reported figures ranging from $23-26 \%$. In contrast, the suggested $40 \%$ primary patency rate at 3 months for the radiological approach was reached in most recent reports. However, should radiologists be proud of a $60 \%$ failure rate at 3 months? With such figures, how can we not fight for better pre-operative mapping and better training of surgeons, leading to creation of more native fistulae, and how can we not demand enforcement of a stenosis detection programme if a graft is eventually placed? How can we not reproach a surgeon who has placed a graft when the radiologist shows evidence that construction of a more durable fistula was possible? 


\section{8 \\ Endovascular Treatment of Native AV-Fistula Thrombosis}

Hannu Manninen

Department of Clinical Radiology, Kuopio University Hospital, Kuopio, Finland

Although early detection and treatment of the stenotic lesions of a failing native AV-fistula is the most secure way to restore the patency of fistula, functional fistula can also be effectively achieved in occasion of thrombosis by endovascular therapy. Primary technical and clinical success rates of about $90 \%$, and $50-70 \%$ primary patency rates at 6 months and $30-50 \%$ at one year can be achieved in freshly thrombosed antebrachial fistulas. Somewhat poorer results are to be expected for upper arm fistulas. The results are well comparable with those of surgical thrombectomy. There are three main access techniques for the endovascular intervention: (1) The intervention and imaging through retrograde access from the antebrachial vein puncture, (2) imaging from the brachial artery through e.g. 22 gauge sheath needle and the intervention through the vein, and (3) the total procedure through antegrade brachial artery puncture. Fistulography through the brachial artery facilitates better visualisation of blood flow dynamics and often complex collateral vessels without placement of blood pressure cuff on the upper arm and inflating the cuff to a suprasystolic pressure, which is usually required for venous technique. Some authors favour routine insertion of retrograde and antegrade venous introducer sheaths, others perform additional antegrade vein puncture only to treat proximal vein lesions. Brachial artery access facilitates the whole intervention in most cases from one puncture but meticulous aftercare is mandatory to avoid puncture site complications. In case of freshly thrombosed AV-fistula with only a short segmental occlusion balloon angioplasty is needed to macerate the thrombus and to treat the underlying stenosis; pressure resistant balloons are preferable and prolonged balloon inflation is often useful in cases with residual stenosis due to organized thrombus and/flow limiting dissections. In long-segment occlusions mechanical thrombectomy may be neded by catheter aspiration or with the use of devices such as rotating minipigtail, Amplatz mechanical thrombectomy device, the Hydrolyser catheter, or Arrow-Trerotola percutaneous thrombolytic device. Pharmacomechanical thrombolysis is needed only seldom, mainly in cases with older thrombosis. Highconcentration urokinase or r-tPA is forcefully injected during the intervention into the thrombotic segment through a pulse-spray type, multiple-sidehole catheter. Self-expanding metallic stents should be reserved for cases with occlusive dissections or vein rupture and they may be useful also to exclude the thrombus in a vein aneurysm by bridging it. Irrespective from the type of the endovascular intervention to restore the patency of thrombosed native $\mathrm{AV}$-fistula, perprocedural heparin of 5,000-7,000 units should be administered and heparinization for at least 24 hours after the intervention is advised.

\section{9}

\section{Strategy: Radiology or Surgery?}

Volker Mickley

Department for Vascular and Endovascular Surgery,

Stadtklinik Baden-Baden, Germany

Introduction: Chronic AV-fistula vein stenoses can be classified according to their respective locations: (type 1) at or close to the arteriovenous anastomosis, (type 2) within the access site, (type 3 ) in the superficial vein upstream the access site. Type 4 stenoses in central (mediastinal or iliocaval veins) will not be discussed here. Different types of stenoses cause different complications and therefore require a differentiated therapeutic approach. Fistula thrombosis in most patients is the consequence of undiagnosed or untreated stenosis. Type 1 Stenoses: Anastomotic venous stenosis is the most frequent long-term complication in distal radiocephalic fistulae. If there is some normal vein between artery and stenosis, anastomotic aneurysm will occur. When after months or years of fistula flow radial artery and cephalic vein are adequately dilated, ligation of the stenotic segment and proximal re-anastomosis is a simple and save procedure giving excellent results. Due to the complex morphology of anastomotic stenoses, interventional treatment is demanding. Often excessive pressures have to be applied to dilate the fibrotic segment, and restenoses are frequent. Prestenotic aneurysm precludes dilatation. Resection of the aneurysm with reconstruction of the artery and proximal reanastomosis is the treatment of choice. Type 2 Stenoses: Long segmental or multiple string-of-bead stenoses of the punctured vein segment are not so easily treated surgically. Either segmental (vein or PTFE) graft replacement or very proximal reanastomosis with loss of considerable access length can be performed. Interventional treatment seems a valuable alternative, because it preserves the access site and does not preclude later surgical revision in case of restenosis. Stent implantation into the punctured vein segment should, of course, not be considered. Type 3 Stenoses: In elbow fistulae, stenoses of the cephalic vein close to its junction with the axillary sometimes cause excessive tortuosity and dilation. Interventional treatment must be performed very carefully to prevent cephalic or axillary vein rupture. Stent implantation into the venous confluence will compromise the deep vein and therefore is contraindicated. When dilatation fails or in case of early recurrence, surgical transposition of the central cephalic vein to the distal axillary vein can solve the problem. Fistula Thrombosis: In most cases of acute thrombosis, careful clinical examination is enough to localise the site(s) of stenosis. Especially in kinking and aneurysmatic veins, surgical thrombectomy (Fogarty catheter and digital thrombus expression) is easier, quicker and less invasive than local thrombolysis. Timely and complete clot removal combined with effective (surgical or on-table interventional) treatment of the underlying stenosis are mandatory for access preservation. 


\section{0 \\ Tutoring and Credentialling in Vascular Access for Hemodialysis}

Salvatore Di Giulio

Department of Nephrology, Dialysis and Urology of S. Camillo Forlanini Hospital, Rome, Italy

Access management for dialysis is a multidisciplinary activity for radiologists, vascular surgeons, nephrologists and dialysis nurses; however clinical practice is often led separately by single 'interventional specialists' (radiologist, nephrologist) or by surgeons. Criteria for validation tutoring and credentialling specialist in interventional procedures are not fully defined. Conflicting results and different reported outcomes in scientific literature could be accounted also by differences in management of vascular access for hemodialysis or lack of adequate interaction among specialists. Outcomes are also influenced by costs and reimbursement policy, monitoring policy of vascular access in dialysis centers, catheters handling, blood flow measurements of fistula and grafts, availability of dedicated rooms for interventional procedures in vascular access. Multiple failures of vascular access management spoil the vascular capital for AV fistulas and grafts, shortening the life expectancy for dialysis patients and results in increased costs. Good clinical practice is possible when levels of competence are defined and respected. Scientific Societies and professional Boards are still defining conditions of tutoring specialists at different levels of clinical experience and credentialling them for interventional procedures according to actual verified experience. Criteria are also needed for evaluation and creditation of referral centers both for training and hospitalization of severe and complicated patients. Moreover need is increasing for updated guidelines and benchmarks for physicians of particular specialty and hospitals in determining the suitability of individuals of each specialty to safely and effectively manage vascular access for hemodialysis. Insurance conditions and legal aspects of interventional procedures are directly related with the definitions of credentialling and tutoring. Databases for vascular access management are also mandatory to assess minimum standards and benchmarks for vascular access management in hemodialysis; databases have also to provide individual follow-up of the 'vascular access record' for individual patients in order to score the actual severity of the patient and the best referral conditions.

\section{Vascular Access Complications}

Chairmen: M. Morosetti, J.H.M. Tordoir

\section{1 \\ Limited Life Expectancy and Vascular Access Strategy for Hemodialysis}

Miltos K. Lazarides

Department of Vascular Surgery, Demokritos University of Thrace, Alexandroupolis, Greece

Traditionally the first choice access in ESRD patients is the wrist radial-cephalic autologous arteriovenous fistula, followed by more proximal access procedures. This position is stated and in NKFDOQI guidelines. The logical concept behind this strategy is the favorable long-term patency of the matured wrist fistulae and the conservation of proximal access sites for future use. However controversy exists regarding the optimal access site in specific subgroups of hemodialyzed patients characterized by their limited life expectancy. Elderly: There is increased interest in this particular subgroup due to the tremendous demographic change within the ESRD patients. It has been estimated that those over 65 years of age comprise more than $60 \%$ of the total number of ESRD patients at present. In those patients parameters such as long-term patency and conservation of proximal access sites are of minimal importance because of their limited life expectancy. Additionally many articles report inferior patency of distal AV fistulae in the elderly [Staramos DN 2000, Miller PE 1999] while other clinical series present inverse results [Colledge $\mathbf{J}$ 1999, Wolowczyk L 2000]. Despite the literature discrepancy, it seems that the initial use of proximal autologous AV upper-arm fistulae or even the more liberal use of synthetic grafts is justified in elderly patients. Diabetics: This category of patients commonly displays medial calcification of the radial artery. The latter when hemodynamically significant prevents the compensatory increase in the arterial flow and therefore the maturation process in distal AV fistulae. The construction of proximal upper-arm AV fistulae as initial approach has been suggested instead [Hakaim AG 1998, Konner K 2000]. Cardiac Failure Patients: Some proximal AV fistulae or AV bridged grafts may carry a flow as high as $3-41 / \mathrm{min}$. This may double the cardiac output at rest, which in marginally compensated patients may lead to congestive cardiac failure. Proximal access sites, wide anastomosis or wide graft diameter should be avoided in cardiac failure patients. Oncologic Patients: This category of patients is particularly susceptible to infections, especially those patients receiving chemotherapy. In the latter group of ESRD patients the use of synthetic $\mathrm{AV}$ bridge grafts should be avoided. Additionally these patients represent difficult candidates for creation of distal AV fistulae due to the tendency of the chemotherapeutic agents to cause thrombophlebitis. Basilic vein transposition is usually an attractive option, as its subfascial location offers protection to this particular vein. 


\section{2}

\section{Blood Flow Reduction in High Output Vascular Access}

Pierre Bourquelot

Clinique Jouvenet, Paris, France

Although a blood flow rate of 300 to $500 \mathrm{ml} / \mathrm{min}$ is sufficient to ensure haemodialysis in adult patients, the actual AVF flow is usually much higher [1]. Apart from proceeding as distal as possible, there is no reliable way to limit blood flow when creating an AVF. The magnitude of AVF blood flow depends of: (1) The initial diameter of the artery, while the role of the initial calibre of the arteriovenous anastomosis remains controversial. As known from congenital AVF, the more proximal is the AVF, the earlier and important is the cardiac overload. (2) The ability of the artery to dilate with time. Among therapeutic AVF, the distal cephalic-to-radial AVF, which has the longest life, is frequently involved in high blood flow. High flow appears infrequently in two kinds of angioaccesses: (a) Distal basilicto-ulnar AVF, probably due to the small diameter of the ulnar artery. (b) Prosthetic arteriovenous bridge-graft, probably because of the early venous anastomosis stenosis. A flow reduction is necessary when flow, related to the body surface area of the patient, is important and/or when a cardiac overload is observed. In cases of associated distal ischemia, flow reduction to decrease vascular steal must be considered as well as the treatment of any artery stenosis, the revascularisation of distal arteries and the ligation of the AVF.

Closure of the Arteriovenous Angio-Access: After a successful kidney transplantation or when it is possible to move to peritoneal dialysis, the closure of the permanent arteriovenous fistula can be done. At the forearm, ligation of the vein near the anastomosis is possible; in case of anastomotic aneurysm, it may be necessary to interrupt the artery. At the elbow, the brachial artery must be spared or patched. In both cases, one must not forget the risk of postoperative hypertension, especially in children. In a few cases, it is possible to close a proximal AVF and to replace it by a more distal permanent access on either of both upper limbs. Obviously in case of multiple fistulas, the ligation technique is also indicated. The popular elbow side-to-side and the perforating vein arteriovenous fistulas deserve many criticisms: retrograde flow to the forearm may induce painful and ischemic distal venous hypertension; perfusion of the profunda brachial veins via the perforating vein will not give any chance of percutaneous venipunctures; the same remark applies to the basilic vein which is not to be punctured without an important risk of brachial artery damages, unless previous surgical superficialization. Arterial distal ischemia, very frequent in diabetics (25\% for J. Elfström) [2], and cardiac overload are the major unrecognised risks of this technique [3]. Closure of the anastomosis straight above the artery is to be done and a new side to end 'only one downstream cephalic or superficialised basilic vein' anastomosis is performed.

Banding: Banding reduction of the venous diameter was the method of choice, but this procedure rarely results in actual flow reduction. Objections to banding are multiple: (1) A peroperative flowmetry is required but prior surgical dissection induces spasm and inaccurate measuring of the flow. (2) Reduction of vascular calibre must be important to avoid the risk of persistent high flow and precise to avoid the risk of thrombosis. Such venous constriction has sometimes been been replaced by a constrictive perivenous mesh prosthesis or by the interposition of a short stepped $(4 \mathrm{~mm}$ to $6 \mathrm{~mm}$ ) PTFE graft. However the degree of reduction in calibre used in both cases is insufficient and no quantitative flow results have been published.

Arterio-Arterial Bypass: In the seventies we gave up early the project to change high flow AVF into arterio-arterial bypass. The theoretical advantage was a marked reduction of basic blood flow, capable to increase highly after cannulation for haemodialysis. The 10 artery-to-artery bypasses which we had created were complicated with early thrombosis, distal artery embolism and painful dialysis due to high-pressure arterial reinjection.

Proximal Artery Ligation: In an end-to-side fistula at the forearm, the major part of the inflow is supplied by the proximal artery, and the remaining comes from retrograde flow through the palmar arch and the distal artery. Ligation of the distal artery was performed by Anderson [4] first for treatment of steal syndrome. We routinely use ligation of the proximal artery [5] for treatment of high blood flow. The surgical procedure is very simple and a $50 \%$ reduction in flow through the AVF can be expected. This method also applies in some cases of proximal high flow AVF coexisting with high bifurcation of the brachial artery which seems to be present in $14 \%$ of individuals. High bifurcation diagnosis is made on the existence of an 'arteriovenous' Doppler signal on both distal forearm arteries (retrograde in one). The AVF, although situated at the elbow, actually involves radial or ulnar artery which, if no angiographic arterial stenosis is present, can be ligated proximally without risk of distal ischaemia. After proximal artery ligation at the wrist, high flow has persisted or reappeared postoperatively in a few cases. Associated ligation of the distal artery, reducing AVF in-flow to small branches from ulnar and interosseous arteries, may be necessary with a $34 \%$ mean reduction rate.

Distal Artery Extension: Distal artery extension involves replacing inflow from brachial artery of a proximal AVF by inflow from one of its smaller calibre distal branches. The original arteriovenous anastomosis is closed at the elbow; an arteriovenous bridge graft is connected between the distal radial or ulnar artery at the forearm and the out-flow tract (vein or rarely prosthesis) at the elbow. A 50\% mean reduction rate is observed. In a few cases, this method may be impossible, due to diabetic distal artery calcifications. Its draw back is eventual future occurrence of a bridge-graft venous anastomosis stenosis.

Radial Artery Transposition: Radial artery transposition, less frequently used, is also a surgical procedure to reduce excessive blood flow through a proximal arteriovenous fistula: the brachial artery which supplies the vein is replaced by the radial artery which calibre is much smaller. For that purpose, the radial artery is first dissected from the forearm, divided at the wrist and then turned upwards to reach the vein at the elbow. Mean reduction rate is $65 \%$. No prosthesis, with its risk of venous anastomosis stenosis, is used. This method seems useful in young patients with normal forearm arteries, specially in children in which bridge-grafts stenosis appears early while long term angioaccess is necessary.

\section{References}

1 Levy BI, Bourquelot P, Ponsin JC, Man NK, Martineaud JP: Noninvasive and invasive blood flowmetry in hemodialyzed patients with high blood flow fistulas. Trans Am Soc Artif Intern Organs 1984;30:335-337.

2 Elfstrom J, Lindell A: Limitations of the use of arteriovenous fistulae in the cubital fossa. Scand J Urol Nephrol 1994;28:123-126.

3 Piza-Katzer H, Laszloffy P, Schidrich R: Complications of antecubital arteriovenous fistula. Vasa 1994:23:163-166. 
4 Anderson CBEEE, Harter HR, Graff RJ, Codd JE: Local blood flow characteristics of arteriovenous fistulas in the forearm for dialysis. Surg Gynecol Obstet 1977; 144:531-533.

5 Bourquelot PCP, Cussenot O: Surgical improvement of high-flow arteriovenous fistulas; in Vascular Access for Hemodialysis (vol 1), edited by Sommer B, Henry ML. Pluribus Press (USA), 1989, pp 124-130.

\section{3}

\section{Ischaemia}

\section{A. Bakran}

University of Liverpool, The Royal Liverpool University

Hospital, Liverpool, UK

Hand ischaemia, usually described as 'steal syndrome', is variously thought to afflict between $1-8 \%$ of patients after arterial venous fistula creation [1-3]. Whilst it is acknowledged that it can occur following a radiocephalic AV fistula formation, it is, however, usually more frequent after an AV fistula which uses the brachial artery as the inflow vessel. Symptoms can vary from complaint of coldness of the hands, cyanosis of fingers, to pain and numbness in the hand and finally to outright loss of sensation with paralysis of hand function (monomelic neuropathy), which is a prelude to ischaemic ulceration and gangrene. Diabetics and those with severe peripheral vascular disease are of special risk of development of steal syndrome. Diagnosis of ischaemia should be based not only on symptoms but also on objective findings. The simplest method of assessment of steal syndrome is the measurement of the wrist brachial pressure index (WBPI) which is similar to the ankle brachial pressure index in patients with peripheral vascular disease of the lower limbs. The WBPI should be at least ' 1 ' in the normal patient prior to fistula creation but if it falls to below 0.6 , then symptoms of steal syndrome seems to occur [4]. This test can be performed on the ward using a simple hand held Doppler device and a sphygmomanometer. Other tests include digital plethysmography and pulse oximetry [5, 6]. Ironically, pressures in diabetics may be inappropriately high due to incompressibility of the diseased arteries in the forearm. All the above are non-invasive tests but arteriography will reveal the extent of steal in such patients, since, in severe cases, very little contrast will be seen to fill the radial and ulnar arteries leading to the hand. The underlying cause of ischaemia is clearly diversion of blood flow up the arteriovenous fistula and away from the hand. Therefore, management of steal syndrome involves mechanisms to reduce the fistula blood flow so that more blood flows distally to the hand. In order to achieve adequate dialysis, a blood flow through a fistula should be a minimal of $500 \mathrm{ml}$ per minute, although brachial fistulae of whatever variety often have flows exceeding 1 litre per minute. Thus, any technique used to reduce fistula flow should reduce blood flow down to a range between 500 and $800 \mathrm{ml}$ per minute and intra-operative method of assessing blood flow should be utilised to confirm adequacy of flow reduction. Techniques used to reduce blood flow are as follows: (1) Avoid a long anastomosis length at time of AV fistula creation since this will increase the risk of excessive blood flow diversion. (2) Banding of autologous or prosthetic graft AV fistulae by (a) plication of fistula using sutures; (b) using a tapered graft segment to interpose between artery and fistula vein; (c) suture a short narrowing segment of PTFE around fistula; (d) DRIL procedure - insert a bypass (vein or prosthetic graft) from brachial artery above the fistula to brachial artery below the fistula and then ligate the brachial artery just distal to the AV fistula anastomosis; (e) closure of the AV fistula in order to salvage the hand in severe cases of ischaemia or instances of ischaemic monomelic neuropathy. The problem with banding is assessing how narrow the segment diameter should be and for what length the fistula should be narrowed. The danger is thrombosis or inadequacy of fistula flow if the surgeon is overzealous. It is, therefore, fundamental that an intra-operative technique is used to assess the adequacy of the flow up the fistula and adequacy of flow reduction to overcome ischaemia. The DRIL procedure involves ligating a normal brachial artery, which may not be the best option for the long term.

In conclusion, ischaemia resulting from an arteriovenous fistula can be a devastating complication for the patient. Assessments of finger blood flow intra-operatively or certainly post-operatively following AV fistula formation will highlight the risks of steal syndrome developing and measures should then be taken early to prevent the long term consequences of this complication.

\section{References}

1 Odland MD, Kelly PH, Ney AL, Anderson RC, Bubrick MP: Management of dialysis associated steel syndrome complicating upper extrematory arteriovenous fistulas: Use of intra operative digital photo plethysmography. Surgery 1991;110:664-667.

2 Rivers SP, Scher LA, Veitch FJ: Correction of steal syndrome secondary to haemodialysis access fistulas: A simplified qualitative technique. Surgery 1992;112:593-597.

3 Bussell JA, Abbott JA, Lim RC: A radial steal syndrome with arteriovenous fistula for haemodialysis. Ann Int Med 1971;75:387-394.

4 Bakran A: Diagnosis and surgical treatment of ischaemia. Dial J 1999; 18(66):259-260

5 Halevy A, Halpern Z, Negri M, Hod G, Weissgarten J, Averbukh Z, Modai $D$ : Pulse oximetry in the evaluation of the painful hand after arteriovenous fistula creation. J Vasc Surg 1991;14:437-439.

6 Dally P, Brantigan CO: Plethysmography and the diagnosis of the steal syndrome following placement of arteriovenous fistulas and shunts for haemodialysis access. J Cardiovasc Surg 1987;28:200-203.

7 Sivanesan S, Bakran A, Howe TV: Characterising flow distribution in AV fistulae for haemodialysis access. Nephrol Dial Transplant 1998;13:3108_ 3110 .

8 West JC, Bertsch DJ, Peterson SL, Gannon MP, Norkus G, Latsha RP, Kelley SE: Arterial insufficiency in haemodialysis access procedures: Correction by 'banding' technique. Transplant Proc 1991;23:1838-1840.

9 Schanzer H, Skladany M, Haimov M: Treatment of angioaccess induced ischaemia by revascularisation. J Vasc Surg 1992;16:861-866.

\section{4}

No abstract received 


\section{5}

Vascular Access and Kidney Transplant

G.P. Segoloni

Chair of Nephrology, Turin University

U.O.A.D.U. Nephrology, Dialysis and Transplantation

Azienda Ospedaliera S. Giovanni di Torino, Turin, Italy

The management of arterio-venous fistulas (AVF) after a successful kidney transplantation represents, in a large majority of cases, a 'clinical grey - zone' where it is difficult to find precise protocols or guidelines. It amazes the scarcity of published papers on this argument and the large variability, often contradictory, of the policies adopted. Different factors, at times submerged, play a role, pros and cons, in this uncertainty of the clinical behaviour: (1) The management of AVF in transplanted recipients, falls, in many cases, in a 'no-man's-land' where each operator (transplant centre and patient's dialytic centre) thinks that it is not his job to pay attention to it. The recipient generally prefer avoid any further surgery and consider his functioning AVF as a spare wheel. (2) A number of AVF undergo a spontaneous closure because of the efferent vein trombosis. Acute rejections episodes or increasing blood viscosity might be involved in this type of local morbidity. (3) On the contrary, still functioning AVF tend, in time, to increase their flow with a sometimes dramatic hypertrophy of the superficial and deep venous system. The artery, due to the atherosclerotic damage, is enlarged and often malacic. The surgical closure of such a AVF demand a skilled operator (arterial reconstruction may be needed), adequate anaesthetic support and could expose the patient to a local (acute phlebitis) and also to a general risk (cardiac arrhythmias at moment of the closure). On the other side, if we take into account that cardiovascular diseases have now emerged among kidney transplant successful recipients as the most important cause of death on the medium-long term, it seems not reasonable to maintain an open AVF after the 1st or 2nd years, if the serum creatinine is below a safety level. In two study (Scorzoni D et al, GIN 1997, van Duijnhoven EC et al, NDT 2001), the only two at my present knowledge, where the effects of the closure of AVF on left ventricular and cardiac function demonstrated a significant improvement, namely, left ventricular end-diastolic diameter and left ventricular mass index significantly $(<0.01)$ decreased. The decrease of the heart rate and the increased of the peripheral vascular resistances, evaluated in the first month, were also significant. In conclusion, it seems reasonable to re-evaluate the policy the management of $\mathrm{AVF}$ in the recipients of a well functioning transplant moving towards an early closure policy when indicated.

\section{Vascular Access Complications}

Chairmen: S. Di Giulio, J. Pengloan

\author{
26 \\ Desperate Vascular Access \\ J osé R. Polo \\ Vascular Access Unit, Hospital General Universitario \\ Gregorio Marañon, Madrid, Spain
}

Unusual vascular access have to be performed in patients without possibility of peritoneal dialysis in which a combination of the following circumstances are present. 1. Obstruction of abdominal or thoracic central veins occasioned by pacemakers, cardiothoracic surgery, previous dialysis catheters etc. 2. Obstruction of upper or lower limb arteries. 3. Severe cardiac failure. Localized subclavian vein occlusion. A branchiojugular graft can be placed at the external or internal jugular vein in cases of subclavian vein occlusion (Haimov M, Surgery 1982;92:109-110; Polo JR et al, Am J Kidney Dis 1990;16:465-468). We have an experience with 54 cases. Periprosthetic infection was very low $(3.7 \%)$ and secondary patency reached almost $60 \%$ at five years. The commonest complication was graftvein stenosis as usual with all kinds of graft. Other alternatives described for intrathoracic vein occlusion had been right atrial bypass (El-Sabrout RA, Duncan JM et al, J Vasc Surg 1999;29:472 478 ) and axillary artery-to-axillary vein grafts (Garcia-Rinaldi et al, Am J Surg 1978;135:265-268; McCann RL, J Vasc Surg 1996;24: 457-462). Axillo-femoral grafts have also been employed (Rueckerman et al, ANNA J 1991;18:567-571). Vena cava occlusion. In absence of arterial pathology a femoro-femoral graft can be placed. A experience with 37 cases and four years $50 \%$ cumulative patency have been reported (Korzets A et al, Nephrol Dial Transplant 1998; 13:1215-1220). In a personal experience with 17 cases, periprosthetic infection was $18 \%$ (infection rate was $8 \%$ in our experience with upper limb grafts) and a high rate of venous stenosis was found. However most of the patients had previous femoral catheters for many time placed to overcome complications in previous upper arm accesses. Subcutaneous transposition of the superficial femoral vein after anastomosis with the distal femoral artery is another. In cases in which arterial pathology discourage a lower limb vascular access a transfermoral permanent catheter of at least $50 \mathrm{~cm}$ in length can be placed. A reasonable experience with 41 of these catheters have been reported (Zaleski GX, AJR 1999;172:493-496). I have experience with three of these catheters used for more than two years. Severe superior and inferior vena cava occlusion. An arterio-arterial graft can be placed (Scholz $\mathrm{H}$ et al, in Henry ML Proceedings of Vascular Access for Hemodialysis VI, Miami, Precept Press, 1999, pp. 255-262). The patients have to be placed on anticoagulation and the flow can be very low in the upper arm (below $200 \mathrm{ml} / \mathrm{m}$ ). Thus, a enlarged dialysis time should be necessary. Other alternatives are translumbar permanent catheters (Lund GB et al, Am J Kidney Dis 1995;25:732-737; Biswal R et al, Cardiovasc Interven Radiol 2000;23:75-78; Azizkhan RG et al, J Pediatr Surg 1992;27:165-169), or transhepatic permanent catheters (Po CL et al, Am J Kidney Dis 1994;24:590-591; Bergey EA, Pediatr Radiol 1999;29:42-45). We have experience with a case of translumbar permcath catheter working for six months with good dialysis flow. 
As the length of femoral catheter is very great a translumbar approach should probably be preferable in cases of inferior vena cava catheters.

\section{7 \\ Biofilm Infections and Antibiotic Resistance

\author{
J. William Costerton \\ Center for Biofilm Engineering, Montana State University, \\ Bozeman, Montana, USA
}

Direct examination of the surfaces of devices used for vascular access, including those used in peritoneal and hemo-dialysis, show very clearly that the bacteria that cause device-related infections grow in matrix-enclosed biofilms. In vitro experiments show that these biofilm populations are 1,000-1,500 times more resistant to antibiotics, than their planktonic counterparts, and in vivo experiments have shown that they are also functionally resistant to host defense mechanisms such as antibodies and phagocytosis. The high level of resistance to antibiotics can now be explained by the observation that biofilm bacteria adopt a profoundly different phenotype, in which the expression of hundreds of genes is altered, and we now know that these agents penetrate biofilms well but have little effect on the altered bacteria. Resistance to host defense mechanisms, on the other hand, appears to be dependent of limited penetration of the voluminous biofilm matrix. These observations agree well with clinical observations to the effect that these device-related infections rarely resolve themselves without intervention, and that antibiotic therapy serves to limit the spread of acute infection and inflammation but almost invariably fails to kill the biofilm bacteria on the surface of the device itself. For this reason, these device-related infections remain chronic and acute episodes occur with increasing regularity. Several strategies for the control of biofilms may be extended to the vascular access field. The first such strategy involves the analysis of gene expression in biofilms formed by key pathogens, to determine which metabolic mechanisms that normally serve as antibiotic targets are missing from these phenotypes. Additionally, new antibiotic targets can now be identified, that are present only in biofilm bacteria, so that new antibiotics can be developed that will target biofilm bacteria specifically. Parallel work with other biofilms has shown that the sensitivity of biofilm bacteria can be rendered very similar to that of planktonic organisms, if the biofilms are exposed to certain frequencies of ultrasound or to very weak DC electrical fields, and these methods are being considered for infection control in vascular access devices. Finally, we have discovered that biofilm formation is controlled by a variety of different chemical signals produced by bacteria, for purposes of population control. These signals, and their close chemical analogues, can now be used to control biofilm formation in industrial and environmental systems, and we propose that they may be equally useful in preventing biofilm formation on medical devices.

\section{8 \\ Permanent Venous Vascular Accesses for Hemodialysis: Catheters and Port Devices}

Bernard Canaud, Hélène Leray-Moragues, Katja Martin, Sébastien Canet

Nephrology, Dialysis and Intensive Care Unit, Dialysis

Research and Training Institute and Lapeyronie University

Hospital, Montpellier, France

Despite significant technical advances over the last two decades, vascular access remains a weak point in the renal replacement therapy. Maintaining a potent vascular access is still a major concern in hemodialysis patient. Vascular accesses related problems represent a primary cause of morbidity and mortality in end stage renal disease (ESRD) patients. It is a permanent source of anxiety having a negative impact on the patients' quality of life. It is a common cause of frustration for physicians and health care providers. It is the main reason for inadequate dialysis delivery being implicated in the morbidity of hemodialysis patient. It is also a major burden for health care financing systems accounting for about $10 \%$ of the budget allocated to ESRD treatment program. Vascular accesses are classified according to their duration of use in two categories: (1) temporary vascular access almost exclusively represented by the central venous catheter that are used up to 90 days; (2) permanent vascular access including tunneled cuffed catheters, native arteriovenous fistula and PTFE prosthesis or autologous graft that are used for more than 3 months. Each vascular access option has its own limitations and specific risks (infection and stenosis for catheter and dysfunction and thrombosis for arteriovenous access). To carry on uninterrupted extracorporeal renal replacement therapy in ESRD patients over several decades, a vascular access armamentarium is required to fulfil needs of the patient's condition. Until recently, the management of vascular access in ESRD patients was exclusively relying on two options: on one side, venous percutaneous catheter with a life expectancy from several weeks to months (temporary and/or permanent catheter); on the other side, arteriovenous fistula with a life expectancy from several years to decade (native or graft prosthesis). In the meantime, no particular vascular option was available for several months to years. In attempt to bridge this gap, subcutaneous port catheter devices have been manufactured. Based on the concept developed for chemotherapy, two vascular access ports (VAP) devices were recently developed (Dialock, Biolink, Middleboro, USA and LifeSite, VascA, Topsfield, USA). These port catheter devices aimed the same objectives, including preservation of patient's comfort, high flow, ease to access and prevention of infections. Initial clinical reports concerning the use of these devices have been positive and encouraging enough to pursue on multicenter trials. Nowadays, blood access for hemodialysis relies on three categories: short term vascular access as temporary catheter; mid term vascular access as permanent catheter and/or catheter port device, long term vascular access as arteriovenous fistula with native vessel or graft interposition. In conclusion, the optimal management of vascular access in hemodialysis patient requires a timely and appropriate insertion of one these options. If we all agree that native arteriovenous fistula is an absolute 'must' for blood access in hemodialysis, we must also recognize that we can't get away from venous accesses. In this perspective, due to its total implantability, port catheter devices offer a new, comfortable and safer venous access for hemodialysis patient. 


\section{9 \\ Long-Term Catheter for Haemodialysis: Early Prescription, Infection Prevention}

\author{
J. Pengloan \\ Unité d'Hémodialyse Chronique, CHU Bretonneau, Tours, \\ France
}

An increasing number of ESRD patients are living with permanent or definitive tunnelled central venous catheters (CVC). However infection is a common and life-threatening complication of CVC. Up to 5.5 bacteremias per 1,000 catheter-days have been reported. Complications are highly frequent and more than $40 \%$ of septic distant locations such as endocarditis, osteomyelitis, epidural abscesses are observed. Mortality is 4-fold higher than in a normal population. In $2 / 3$ of the cases the causative pathogens were staphylococci and in $2 / 3$ of the cases the $\mathrm{CVC}$ had to be removed. A rigorous strategy of prevention and early treatment is the best way to prevent these severe complications. It is based on the understanding of the mechanisms of the catheter-related infection which spreads out through 4 steps: introduction into the catheter, adhesion, multiplication in a protective biofilm and dissemination of the bacteria in the bloodstream. The introduction of bacteria is firstly dependent on aseptic handling of CVC. Early detection of intraluminal colonisation can be detected by routine monthly culture of the content (i.e. heparin) aspirated from each lumen of CVC. Treatment is instituted in case of confirmed intraluminal colonisation, and must be immediately started in case of fever; it is based initially on antistaphylococcal antibiotics; antibiotics having the least risk of bacterial resistance and easy to prescribe in hemodialysis patients have to be chosen. Antibiotic treatment is associated with fibrinolytic treatment and antibiotic-lock. In our experience (64 permanent tunnelled silicone $\mathrm{CVC}$ ) aseptic connect and disconnect procedures associated with early detection and treatment of intraluminal contamination resolved in a low rate of catheter-related infection ( 0.6 per 1000 catheter-days) and a good actuarial survival for CVC (70\% at three years); one CVC only had to be removed for infectious cause. Treatment was based on the association of Cefazolin ${ }^{\circledR}$, Netilmycin ${ }^{\circledR}$ and Urokinase ${ }^{\circledR}$. Other preventive methods have been proposed: treatment of carriage of staphylococcus aureus, antibiotic/antiseptic bounded catheters, preventive antibiotic-lock, citrate-lock, subcutaneous ports. However several important points have to be focused on: rigorous asepsis, early detection and treatment of colonisation or bacteremia, reservation of Vancomycin ${ }^{\circledR}$ as a second line treatment and only for methicillinresistant staphylococcus.

\section{0 \\ Catheter Dysfunction: Diagnosis and Treatment}

Francesco Quarello, Giacomo Forneris, Marco Pozzato

Nephrology and Dialysis Unit, San Giovanni Bosco Hospital, Turin, Italy

In recent years tunneled central venous catheters have come to be an important tool in the management of patients requiring hemodialysis, but their possible malfunction still represents an important drawback. This event not only may cause the delivery of an insufficient dialytic dose, but also herald a serious complication of the central venous system. Catheter dysfunction can either arise immediately after vein cannulation or later on. In the former cases a misplacement of the catheter at the time of insertion has to be excluded. Catheter kinking, sometimes difficult to ascertain fluoroscopically without contrast medium, and improper tip placement, critically important with some kind of devices, are other possible causes for dysfunction. In the absence of fluoroscopic or electrocardiographic guidance the occurrence of malfunction due to incorrect position is more frequent. The failure to achieve a continuous aspiration or an adequate blood flow may stem from a partial or complete thrombosis of the catheter. Insufficient flushing of the catheter with anticoagulant locking solution and leak of fluid through the side holes may favour the thrombosis. Intraluminal thrombosis is the most frequent cause for malfunction and is currently treated, when aspiration fails to reestablish the patency of the catheter, through the infusion of fibrinolytic agents (urokinase 5,000-10,000 IU/ml), according to different protocols, that proved to be efficacious in $75-95 \%$ of the cases. Alteplase (TPA) at the dose of $2 \mathrm{mg}$ is a promising alternative to urokinase. If the locking method fails to fully restore catheter patency, a periluminal thrombosis may be responsible for the malfunction. The fibrin sheath sleeve that surrounds the catheter can be effectively treated with infusion of increasing doses of urokinase $(20,000-$ $250,000 \mathrm{IU})$. A persistent malfunction can be overcome by exchanging the catheter over a guidewire, taking into account the risk of placing the new catheter back into a retained sheath is high. On the other hand, a fibrin sheath stripping using a snare catheter, successful in $92-98 \%$ of the cases, can be effectively used to solve the problem; however, an experienced interventional radiologist is needed and the recurrence of the problem is not precluded. Mural or atrial thrombi usually require the removal of the catheter and the institution of a systemic anticoagulation. Oral anticoagulant drugs as preventive measure are currently widely adopted, even if data in support of their use are still lacking. From the diagnostic point of view phlebography and echocardiography are the most effectively used tools. Angio-CT too has recently been utilized with interesting results.

\section{1 \\ Central Venous Catheters and Factors Predicting Dysfunctions, Duration and Outcome \\ F. Galli, V. Piazza \\ S. Maugeri Foundation, Pavia, Italy}

Among haemodialysis vascular accesses, permanent central venous catheter carries the highest rate of complications; for that reason catheter should be considered only in very selected cases as permanent vascular access. As a matter of fact the duration of the catheter is directly related to the number of complications occurred between insertion, removal and/or exchange; besides catheter complications can affect either the quality of dialysis given to the patient or, under certain circumstances, the patient's life. Catheter complications are usually divided into those following insertion and those related to management. The former ones must be absent or minimised, in order to ensure a good start of the device; so the vein chosen for catheter 
insertion must be correctly and exactly cannulated (by means of ultrasound guided puncture), the catheter tip must be placed in the most proper site (by means of fluoroscopy or endocavitary electrocardiography) and finally the catheter must be suitably anchored and the device must be comfortably applied to the patient. As regards management complications, basically thrombosis and infection, a low rate must be sought to achieve good duration and performance of the device. The pathogenetic mechanisms of thrombosis are not completely understood, so at present it is difficult to adequately prevent thrombotic events, considering that, nowadays, all utilised catheters are supposed to be of good quality. Anticoagulant oral therapy has been demonstrated to reduce the rate of thrombotic events; also monthly prophylactic cleaning with low doses of urokinase seems to have a positive effects, but prospective and controlled studies are lacking. As regards infection, the best prophylactic approach is the strict adherence to protocol of use and medication of the catheter, stated by each dialysis unit. The antibiotic therapy is aimed at curing the patient and possibly at preventing catheter removal; the more early and accurate the diagnosis the more effective the therapy. This is the reason why a close co-operation with the bacteriology laboratory is mandatory. Systemic antibiotic therapy can obtain better results in order to preserve the catheter if associated with the antibiotic lock therapy. Another complication of catheter placement, not related to the above-mentioned ones, has been observed in almost all central venous catheters: the so called 'fibrin sleeve', which can be considered a rejection to a foreign body, i.e. the catheter. The sheath surrounds the whole catheter, both in the endoluminal and extraluminal sites; it is composed not only by fibrin but it is made up also of a true tissue, the reactive response of the body to a foreign element. Limiting and possibly blocking the fibrin sleeve formation is a challenge for the next years. In order to achieve this improvement, catheters will be constructed with more hemocompatible materials but new therapeutic strategies will be needed to induce a tolerance to the foreign body, the catheter. Anyway, from now on, we must consider central venous catheter not as a separate element, but as a part of a system, the patient-catheter system, to be studied as a whole.

\section{2}

\section{Stenosis and Thrombosis of Central Veins}

Volker Mickley

Department for Vascular and Endovascular Surgery,

Stadtklinik Baden-Baden, Germany

Introduction: Central venous obstruction arising from longterm or repeat catheterizations for haemodialysis access is a frequent problem in ESRD patients. If the draining vein of a functioning vascular access is obstructed, venous hypertension with arm swelling, pain and ulceration will occur. Surgery (patch angioplasty or bypass procedures) can be difficult and hazardous in these often multimorbid patients. Thus it seems necessary to define the relative value of interventional treatment (percutaneous angioplasty and stent implantation) in comparison to surgical reconstruction of central venous obstructions in haemodialysis patients. Patients and Methods: From 1993 through 2000, 37 haemodialysis patients (3\% of all access procedures, $5 \%$ of all access patients) presented with symptomatic mediastinal vein obstructions (disabling arm swelling in 37, distal ulcerations in two) on the side of a functioning access. Percutaneous angioplasty and stent implantation was possible in 23 patients $(62 \%)$. When interventional procedures were unsuccessful, venovenous bypasses were implanted (6 patients, $16 \%$ ), or access ligation was performed ( 8 patients, $22 \%$ ). All patients were followed up prospectively. Results: Following stent implantation one asymptomatic pulmonary stent embolism (4\%) and two stent misplacements (9\%) were documented. The latter were successfully treated with another stent. In the surgical group, one patient died at eight weeks due to late complications of a cephalosporine-associated Lyell syndrome. Patency rates (life table-method) were not significantly different among the groups:

\begin{tabular}{llllll}
\hline Patency & \multicolumn{2}{c}{ Primary, years } & & \multicolumn{2}{c}{ Secondary, years } \\
\cline { 2 - 3 } \cline { 5 - 6 } & 1 & 2 & & 1 & 2 \\
\hline Stents, \% & 76 & 61 & & 96 & 87 \\
Bypasses, \% & 83 & 67 & & 100 & 83 \\
\hline
\end{tabular}

Conclusion: For central venous stenoses in haemodialysis patients, angioplasty and stent implantation can be recommended as a safe and durable means to restore patency. Still there is a role for bypass surgery in severely symptomatic central venous occlusions when stent implantation is impossible or has failed. In high-risk patients, however, access ligation should be performed. 
Bagolan, P. 9

Bakran, A. 10, 23

Barbosa, J. 14, 24

Berardinelli, L. 6

Bourquelot, P. 5, 22

Canaud, B. 28

Canet, S. 28

Cavatorta, F. 12

Costerton, J.W. 27

Di Giulio, S. 20

Dionisio, P. 12

Ervo, R. 12

Fabbrini, G. 9

Forneris, G. 30

Galli, F. 31

Galli, S. 12

Günther, R.W. 16

Haage, P. 16

Lazarides, M.K. 21

Leray-Moragues, H. 28
Malovrh, M. 3

Manninen, H. 18

Martin, K. 28

Medici, L. de 15

Mickley, V. 19, 32

Morosetti, M. 8

Pengloan, J. 2, 29

Piazza, V. 31

Pieri, S. 15

Polo, J.R. 7, 26

Pourchez, T. 1

Pozzato, M. 30

Quarello, F. 30

Raynaud, A. 4

Segoloni, G. 25

Stortoni, F. 5

Tordoir, J.H.M. 11, 13

Turmel-Rodrigues, L. 17

Vorwerk, D. 16

Zollo, A. 12 